\title{
1 Complement facilitates developmental microglial 2 pruning of astrocyte and vascular networks
}

3

Gopalan Gnanaguru ${ }^{1, ~}$, Steven J. Tabor ${ }^{1}$, Kentaro Yuda ${ }^{1}$, Ryo Mukai $^{1 \#}$, Jörg Köh2²,3, Kip M. Connor ${ }^{1 \#, *}$.

${ }^{1}$ Angiogenesis Laboratory, Department of Ophthalmology, Massachusetts Eye and Ear Infirmary, Harvard Medical School, Boston, MA, USA 02114

${ }^{2}$ Institute for Systemic Inflammation Research, University of Lübeck, Lübeck, Germany 23562

${ }^{3}$ Division of Immunobiology, Cincinnati Children's Hospital Medical Center and University of Cincinnati College of Medicine, Cincinnati, $\mathrm{OH}, \mathrm{USA}$

\# Present address:

KMC: Head of Ophthalmology Research Unit, Biogen, Cambridge MA 02142

RM: Gumma University Graduate School of Medicine, Department of Ophthalmology, Showa-cho Maebashi Japan 371-8511

To whom correspondence should be addressed.

*Email: gopalan_gnanaguru@meei.harvard.edu and Kip.Connor@gmail.com 
Abstract

Microglia, the resident immune cell of the central nervous system, play a pivotal role in facilitating neurovascular development through mechanisms that are not fully understood. This current work resolves a previously unknown role for microglia in facilitating the developmental pruning of the astrocytic template resulting in a spatially organized retinal vascular bed. Mechanistically, our study identified that local microglial expression of complement (C)3 and C3aR is necessary for the regulation of astrocyte patterning and vascular growth during retinal development. Ablation of retinal microglia, loss of C3 or C3aR reduced developmental pruning and clearance of astrocytic bodies leading to increased astrocyte density leading to altered vascular patterning during retinal vascular development. This data demonstrates that $\mathrm{C} 3 / \mathrm{C} 3 \mathrm{aR}$ signaling is an important checkpoint required for the finetuning of vascular density during neuroretinal development. 
62

\section{Introduction}

High-energy demanding tissues, including neural tissues, require the development of a complex vascular network for their growth, survival, and function. The process of vascular development in neural tissue is orchestrated through highly regulated crosstalk between cell types such as neurons, glial cells, and vascular cells for the establishment of functional blood vessels that contain blood neural barrier properties (Abbott et al., 2006; Fruttiger, 2007; Langen et al., 2019). We now know that the resident immune cell of the central nervous system (CNS), microglia, is a vital contributor in promoting neural-vascular development (Fantin et al., 2010; Rymo et al., 2011). However; the mechanism(s) through which microglial cells dictate vascular growth in the CNS is not fully understood. To further characterize the role of microglia in vascular development, we utilized the retina as a model system allowing us to explore the cellular and molecular contributions of microglia in forming this complex network.

The development of the vascular system in the retina is regulated by non-resident cell types, microglia that populate the retina during early embryonic stages (Santos et al., 2008) followed by astrocytes that enter the retina through the optic nerve head around birth in mice (Fruttiger et al., 1996; Stone and Dreher, 1987; Watanabe and Raff, 1988). Astrocyte migration and template formation in the retina is an essential step for vascular development and interestingly species that lack astrocytes in the retina remain avascular (Schnitzer, 1988; Stone and Dreher, 1987; Zhang and Stone, 1997). In the vascularized retina, ganglion cell derived Pdgfa acts as a chemoattractant that 
87 stimulates astrocyte entry into the retina at the time of birth (Fruttiger et al., 1996;

88 Fruttiger et al., 2000). In response to Pdgfa, astrocytes proliferate and migrate in a centrifugal fashion to form a spatially organized template (that completes around postnatal day $(P)-5$ thereby allowing subsequent vascular growth to occur (Fruttiger,

91 2007; Fruttiger et al., 1996; Fruttiger et al., 2000; Gnanaguru et al., 2013). While the

92 vascular template is forming and rearranging, the leading endothelial tip cells follow the

93 astrocyte laid template (beginning at P0) to form the superficial vascular layer by P7

94 (Dorrell et al., 2002; Gerhardt et al., 2003), followed by the formation of an

95 interconnected deep and intermediate vascular layers that completes around P15

96 (Fruttiger, 2007). Intriguingly, disruption of astrocyte template formation perturbs

97 subsequent vascular growth (Edwards et al., 2010; Gnanaguru et al., 2013; Tao and

98 Zhang, 2016). Although the importance of astrocyte migration into the retina for

99 vascular growth is known, it remains unclear what signaling mechanism(s) modulate the 100 spatial organization of astrocyte and vascular networks during retinal development. In

101 cases of perturbed or abnormal vascular growth, permanent vision loss can occur

102 (Hartnett, 2020; Holmstrom et al., 2007; Wong et al., 2016), thus understanding these

103 mechanism(s) of vascular development could lead to new strategies for treating retinal 104 pathologies.

105

106 Microglia precede astrocytic entry into the neuroretina (Santos et al., 2008) and are 107 known to contribute to vascular growth by facilitating endothelial tip cell anastomoses 108 (Fantin et al., 2010; Rymo et al., 2011). However, to date it was less clear if microglia 109 also interact with astrocytes to assist astrocytic template formation. During neural 
110 development microglia play an indispensable role in the formation of a defined and

111 functional neural circuitry mediated in part through microglia and the complement

112 signaling system (Anderson et al., 2019; Paolicelli et al., 2011; Schafer et al., 2012).

113 The complement system is an integral component of the innate immune system, initially

114 discovered as a component involved in opsonization and clearance of foreign

115 pathogens (Holers, 2014). Recent studies imply that in addition to its immune response

116 function, the complement system regulates wide-variety of biological functions such as

117 synaptic pruning, regulation of glial-microglial crosstalk, clearance of abnormal

118 neovascular growth, removal of stressed or dying cells and modulation of cell

119 metabolism (Anderson et al., 2019; Baudino et al., 2014; Coulthard et al., 2018; Inafuku

120 et al., 2018; Kim et al., 2016; Lian et al., 2016; Mevorach et al., 1998; Mukai et al.,

121 2018; Roy et al., 2013; Schafer et al., 2012; Sweigard et al., 2015). Depending on the

122 context/stimuli, complement activation is initiated through classical, alternative, or lectin

123 pathways (Pitulescu et al.), all of which lead to cleavage of C3 and C5 fragments, i.e.

124 the large C3b and C5b and the small C3a and C5a fragments (Holers, 2014; Merle et al., 2015). In particular, the release of cleaved C3a binding and activation of its cognate

126 C3aR regulates diverse biological functions such as immune cell recruitment,

127 development of the cerebellum, modulation of embryonic neural progenitor proliferation

128 and cognitive performance, chick eye morphogenesis, and astrocyte-microglial crosstalk

129 to elicit inflammatory response in neurodegenerative conditions (Benard et al., 2008;

130 Coulthard et al., 2018; Grajales-Esquivel et al., 2017; Hartmann et al., 1997; Ingersoll et

131 al., 2010; Lian et al., 2016; Litvinchuk et al., 2018). Potentially, suggesting a more

132 ubiquitous role of $\mathrm{C} 3 \mathrm{a} / \mathrm{C} 3 \mathrm{aR}$ signaling during retinal vascular development. 
134 Prior to superficial retinal vascularization, the astrocyte template undergoes

135 rearrangement and a significant number of astrocytes are eliminated through an

136 unknown mechanism (Chan-Ling et al., 2009; Punal et al., 2019). Given the role of C3

137 in synaptic pruning and microglial-astrocyte crosstalk (Cong et al., 2020; Lian et al.,

138 2016; Schafer et al., 2012), we hypothesized and tested if C3 facilitates microglial

139 pruning of the astrocytic template required for astrocyte spatial organization and

140 subsequent vascular network development.

142 To assess the role of microglia in the regulation of the astrocytic and endothelial

143 networks formation during retinal development we utilized an antibody against the

144 purinergic receptor, P2ry12, which is microglial specific (Butovsky et al., 2014). We then

145 depleted microglia using colony-stimulating factor-1 (Csfr1) antagonist (Hilla et al.,

146 2017; Okunuki et al., 2019; Okunuki et al., 2018; Valdearcos et al., 2014), in order to

147 investigate the significance of microglial loss on astrocyte template formation and

148 subsequent vascular development. Data showed that the depletion of microglia severely

149 disrupted astrocytic template patterning and subsequent vascular growth. To

150 understand the mechanism of microglial regulation of astrocyte template patterning, we

151 examined C3 tdTomato and C3aR tdTomato expressing murine retina, as C3a/C3aR

152 signaling is involved in microglia/astrocyte crosstalk (Lian et al., 2016). Results revealed

153 concomitant expression of $\mathrm{C} 3$ and $\mathrm{C} 3 \mathrm{aR}$ in microglia that are closely associated with

154 astrocytes in the vascularizing and adjacent avascular area. Finally, deficiency of C3 or 
155 C3aR and functionally blocking C3aR illustrated the importance of complement

156 mediated pruning by microglia for the proper development of the astrocytic template.

\section{Results}

Microglia immigrate into the retina during embryonic development and closely

development.

162 To examine the distribution of microglia prior to astrocyte and endothelial entry into the

163 retina, we assessed E13.5 Cx3cr1-egfp retinas with a microglial specific P2ry12 marker.

164 Analysis showed that P2ry12 and Cx3cr1-egfp double positive microglia were localized

165 within the developing ganglion cell layer as well as in the neuroblastic layer (Figure

166 S1A). Since endothelial tip cell migration and vascularization in the retina is entirely

167 dependent on the prior formation of the astrocytic template (P0-P5) (Edwards et al.,

168 2010; Fruttiger et al., 1996; Gnanaguru et al., 2013; Stone and Dreher, 1987; Tao and

169 Zhang, 2016; Uemura et al., 2006), we examined if microglia closely associate with

170 astrocytes during astrocytic template formation (P0) and rearrangement (P5) allowing

171 for subsequent endothelial tip cell migration and vascular patterning.

Analysis of P0 retinal flatmounts revealed the close association of microglial ramified

174 processes with the developing astrocytic template as well as the entering endothelial tip

175 cells around the optic nerve head $(\mathrm{ONH})$ region (Figure 1A). In addition, at P0, we

176 noticed the enveloping of microglial processes over astrocytes that are beginning to

177 form a naïve template in the central and peripheral retinal regions required for 
178 subsequent vascular growth (Figure 1A). Further developmental analysis at P5

179 illustrated the existence of intimate interactions between microglia, astrocytes, and

180 endothelial tips cells in the vascularized regions and microglia-astrocyte interactions in

181 the avascular regions (Figure 1B). Of note, P2ry12 labeled retinal microglia exhibit

182 more prominent structures as compared to staining with lba1 or isolectin B4 (Figure 1C,

183 1D, and Figure S1B), which allowed us to assess a clearer role for these cells than

184 previously known (i.e., astrocytes and endothelial cells) during vascular development.

Depletion of microglia is associated with increased astrocytic density and spatial

187 patterning

188 To further investigate the functional relevance of microglial interactions with astrocytes

189 and endothelial cells during the critical steps of astrocytic template assembly and

190 subsequent vascular development, we designed a strategy to deplete microglia from

191 P0. Colony-stimulating factor 1 receptor (Csf1r) signaling is required for microglial

192 survival (Elmore et al., 2014; Okunuki et al., 2019; Okunuki et al., 2018), and prior work

193 found that Csf1r deletion during early development is embryonic lethal (Chitu and

194 Stanley, 2017), thus we utilized a pharmacologic approach whereby Csf1r is inhibited to

195 deplete microglia (Okunuki et al., 2019; Okunuki et al., 2018). To overcome the

196 technical challenges to deplete microglia from birth, timed-pregnant C57BL6J females

197 were fed a diet incorporated with Csf1r specific antagonist (PLX5622) from

198 E13.5/E14.5. We have recently reported that mice fed a diet containing Csf1r specific

199 antagonist (PLX5622) more effectively depletes microglia in the retina, similar to genetic 
200 approaches, but with minimal off-target effects (Okunuki et al., 2019; Okunuki et al., 201 2018).

203 P2ry12 immunostaining of P1 and P5 retinal flatmounts of litters from control diet fed 204 group or PLX5622 diet fed group show that the PLX5622 antagonist successfully 205 depleted microglia in comparison to controls (Figure S2A and S2C). Analysis of RNA extracted from P1 and P5 retinas of PLX5622 treated animals also showed significant

207 decrease in Csf1r, Tmem119, and P2ry12 gene expression levels in comparison to the 208 P1 control treated group (Figure S2B and S2D). Flowcytometric analysis further confirmed significant microglial depletion (Figure S3C and S3D). Following microglial

210 depletion during early retinal vascular development, we examined P1 (active astrocyte

211 template assembly phase) and P5 (active astrocyte template rearrangement and

212 vascular growth phase) retinas of litters from control mice and in mice lacking microglia.

214 Although microglial depletion did not prevent astrocyte migration into the retina, analysis

215 of P1 retinal flatmounts from pups lacking microglia showed a moderate reduction in the

216 astrocytes migrated area compared to control retinas (Figure S2E). While at P5, control

217 retinal flatmounts displayed typical astrocyte honeycomb patterning in the central

218 vascular and peripheral avascular retina (Figure 2A). In contrast, examination of litters

219 from mice lacking microglia displayed abnormal clustering of astrocytes in the central

220 vascular region (Figure 2A). In the peripheral avascular region, spatial distribution of

221 astrocytes was dramatically increased in pups lacking retinal microglia compared to

222 controls (Figure 2A and 2C). Furthermore, quantitative analysis by flowcytometry 
223 showed that the percentage of Pdgfra+ (a marker for astrocytes) astrocyte population

224 was significantly increased in pups lacking microglia in comparison to the control group

225 (Figure 2D, 2E, and S3B) with no aberrant changes in expression of the retinal

226 astrocytes growth factor Pdgfa (Fruttiger et al., 1996; Fruttiger et al., 2000) (Figure

227 2B). Intriguingly, the abnormal astrocytic density and clustering phenotype persisted

228 even following the completion of vascular development at P15 (Figure S4). Astrocyte

229 distribution at P15 retinal flatmounts with and without microglia were assessed using the

230 Gfap marker as it is strongly expressed in mature astrocytes (Chan-Ling et al., 2009).

231 Gfap immunostained P15 retinas of pups lacking microglia showed significant increase

232 in astrocyte distribution along with increased astrocytic template branching in the central

233 and peripheral retinas compared to controls (Figure S4A and S4B). P15 retinal

234 flatmounts that lacked microglia also contained abnormal clustering of astrocytes

235 compared to control retinas (Figure S4C). Taken together, these findings strongly

236 implicate microglia as playing a significant role in supporting the spatial establishment of

237 the astrocytic template required for retinal angiogenesis.

Depletion of microglia reduced subsequent astrocyte dependent vascular growth

240 and density

241 Proper formation of the astrocytic template and microglia derived signaling are

242 necessary for endothelial tip cell migration and subsequent formation of the superficial

243 retinal vascular network (Edwards et al., 2010; Gerhardt et al., 2003; Gnanaguru et al.,

244 2013; Outtz et al., 2011; Rymo et al., 2011; Tao and Zhang, 2016). As microglial

245 depletion resulted in defective assembly of the astrocytic template, we next examined 
246 the effects on subsequent vascular growth (Figure S5). Analysis of retinal flatmounts in

247 litters from mice lacking microglia showed that the vascularized area at P1 and P5 were

248 significantly reduced in comparison to litters from control group (Figure S5A and S5B).

249 We further confirmed that the PLX5622 treatment did not alter gene expression levels of

250 vegf isoforms, key for retinal vascular growth (Figure S3E). Consistent with the

251 previous findings (Fantin et al., 2010; Rymo et al., 2011), depletion of microglia also

252 significantly reduced vascular density and branching points, while dramatically

253 increasing lacunarity in P5 litters of mice lacking microglia compared to litters of control

254 animals (Figure S5C and S5D). Examination of P15 retinal flatmounts of litters from

255 mice lacking microglia revealed that the vascular phenotype observed (reduced density,

256 branching points, and increased lacunarity) persisted even after the completion of

257 vascular development (Figure S6).

258

Microglia utilize complement to facilitate spatial astrocytic template for subsequent vascular development

261 During retinal vascular development, large numbers of astrocytes are eliminated

262 through an uncharacterized mechanism likely facilitated by microglia peaking at P5

263 (Chan-Ling et al., 2009; Punal et al., 2019), to help spatially organized astrocytic

264 template establishment. In agreement with the previous findings (Chan-Ling et al., 2009;

265 Punal et al., 2019), analysis of P5 retinal flatmounts showed engulfed Gfap positive

266 astrocytic cellular debris localized within the microglial endosomal/lysosomal membrane

267 protein CD68 (Figures 3A, 3A' and Figure S7A). 
269 C3 derived signaling is well known to facilitate the refinement of neural architecture by

270 pruning and eliminating dying neurons (Anderson et al., 2019). Therefore, we

271 investigated if C3 regulates microglial pruning of the astrocytic template for the

272 establishment of a defined spatially organized vascular network. Examination of C3

273 transcript levels during the active astrocytic template rearrangement phase (P5) showed

274 significant increase relative to P0 (early astrocytic template assembly phase) and P15

275 (vascular development completion phase) (Figure 3B). Moreover, assessment of P5

276 retinal flatmounts using C3 tdTomato reporter mice showed robust localization of C3 in

277 microglia that are closely associated with astrocytes in the vascular growth and adjacent

278 avascular zones at active astrocyte template rearrangement and subsequent vascular

279 developmental phase (Figure 3C and 3D).

281 We next assessed P5 retinal flatmounts of C3 deficient mice along with their WT

282 littermate controls to define how the loss of C3 affected astrocytic template

283 rearrangement. Analysis of C3 deficient P5 retinas showed a significant reduction in the

284 localization of Gfap positive astrocytic cell debris within microglial CD68 compared to

285 WT littermate control retinas (Figure 3E and Figure S7B). Notably, in comparison to

286 the WT P5 littermate retinal flatmounts, analysis of Pdgfra immunostained P5 C3

287 deficient retinal flatmounts displayed a significant increase in astrocyte template density,

288 branching index, and decreased lacunarity, indicative of reduced spatial gap in

289 astrocyte template (Figure 3F). 
291 Given that the loss of C3 dramatically increased astrocytes density and spatial

292 patterning (Figure 3F), we next analyzed the effect of C3 loss on subsequent vascular

293 growth at P5. Data show that in comparison to WT littermates, P5 C3 deficient retinal

294 flatmounts showed significant increase in the vascularized area (Figure S8A). In

295 addition to increased vascular growth, vessel density was significantly increased in the

296 P5 retinal flatmounts of C3 deficient pups compared to P5 WT littermate retinal

297 flatmounts (Figure S8B), while the spatial gap was significantly reduced in P5 C3 KO

298 compared to WT littermate controls (Figure S8B). These results suggest that C3 plays

299 an important role in regulating spatial astrocytic template assembly for subsequent

300 superficial retinal vascular development.

301

C3aR activation controls microglia-mediated phagocytosis of astrocytes

$303 \mathrm{C} 3 \mathrm{a} / \mathrm{C} 3 \mathrm{aR}$ signaling has been shown to regulate microglia-astrocyte crosstalk (Lian et

304 al., 2016; Litvinchuk et al., 2018). Therefore, we investigated if microglia use a

$305 \mathrm{C} 3 a / C 3 a R$ dependent signaling mechanism to phagocytose and eliminate astrocytic

306 bodies leading to a spatially organized astrocyte network during vascular development.

307 During the astrocytic template rearrangement phase, expression analysis of C3aR

308 revealed a significant increase at P5 in comparison to P0 and P15 retinas (Figure 4C).

309 Further examination of P5 retinal flatmounts of C3aR-tdTomato reporter mice (Quell et

310 al., 2017) showed strong expression of C3aR in microglia within vascular growth and

311 avascular zones (Figures 4A and 4B). In order to test if C3aR is involved in the

312 phagocytosis and elimination of astrocyte bodies, we isolated and cultured retinal

313 microglia and astrocytes from P5 pups. After labeling cultured astrocytes with Dil to 
314 track and identify this population, astrocytic cell death was induced by staurosporine

315 treatment. Retinal microglia were treated with Dil-labeled dead astrocytic bodies in the

316 presence or absence of C3aR neutralizing antibodies or control IgG to determine if

317 C3aR facilitates microglial phagocytosis of astrocytic dead cell bodies. Blocking of

318 C3aR significantly reduced microglial engulfment of Dil-labeled dead astrocyte bodies

319 when compared to IgG control (Figure 4D). Examination of P5 C3aR KO retinal

320 flatmounts showed a significant reduction in the localization of Gfap positive astrocytic

321 cell debris within microglial CD68 compared to WT littermate control retinas (Figure

322 4E). Analysis of Pdgfra+ astrocytic template formation at P5 also showed increased

323 astrocytic density and branching, and reduced spatial distribution in $\mathrm{C} 3 \mathrm{aR} \mathrm{KO}$ retinas

324 compared to WT littermate controls (Figure 4E). Astrocytic template dependent

325 subsequent superficial vascular growth and vascular density were increased with

326 reduced spatial vascular patterning in $\mathrm{P} 5 \mathrm{C} 3 \mathrm{aR} \mathrm{KO}$ retinal flatmounts in comparison to

327 its WT littermate controls (Figures S8C and S8D). These results illustrate that

328 microglial C3aR receptor facilitates the developmental elimination of astrocytes for

329 spatial patterning and for organized succeeding vascular network formation.

331 In summary, these results identify microglia as a key regulator of astrocyte spatial

332 patterning and subsequent vascular development through the $\mathrm{C} 3 \mathrm{a} / \mathrm{C} 3 \mathrm{aR}$ derived

333 signaling axis. 
337 Microglia are essential for neural development (Anderson et al., 2019; Santos et al.,

338 2008; Schafer et al., 2012), and in particular microglia play an important role during

339 neurovascular growth (Fantin et al., 2010; Rymo et al., 2011). In this study, we

340 uncovered a mechanism through which microglia direct neurovascular development in

341 the retina. Results from this study show that the depletion of microglia early during

342 astrocytic patterning and subsequent vascular growth phases increased astrocyte

343 density and caused abnormal astrocyte template formation and reduced vascular

344 growth (Figure 2, Figures S3, S4, S5, and S6). Mechanistically, our study identified

345 that microglia derived $\mathrm{C} 3 \mathrm{a} / \mathrm{C} 3 \mathrm{aR}$ signaling regulates spatial patterning of astrocyte and

346 vascular networks (Figures 3, 4, and figure S8). Specifically, deficiency of C3 resulted

347 in dramatic reduction in microglial phagocytosis of astrocyte debris leading to increased

348 astrocyte density and reduced spatial gap (Figure 3). In addition, loss of C3aR

349 diminished microglial phagocytosis of dying astrocyte bodies (Figure 4), implicating a

350 role for the $\mathrm{C} 3 \mathrm{a} / \mathrm{C} 3 \mathrm{aR}$ signaling axis in eliminating astrocytes during retinal

351 development. Taken together, these findings uncovered a new functional role for

352 microglia derived C3 signaling in regulating retinal vascular development.

354 Recent advancements in the discovery of microglial specific markers, such as P2ry12,

355 which are highly expressed during development (Butovsky et al., 2014), allowed us to

356 further define the extensively ramified morphology of microglia during development

357 along with their close interaction with astrocytes during development. The finding of

358 elaborate ramified morphology of microglial associations with astrocytes and endothelial

359 cells during development is particularly intriguing, as prior use of generic myeloid cell 
360 markers such as Iba1 did not reveal the complex morphology or the extent to which

361 microglia associate with astrocytes during development (Fantin et al., 2010; Outtz et al.,

362 2011; Rymo et al., 2011). Given the fact that formation of the astrocyte template is the

363 most critical step for retinal vascular development (Fruttiger, 2007; Fruttiger et al., 1996;

364 Uemura et al., 2006), previous studies provided insights into the microglial regulation of

365 endothelial tip cell fusion during retinal vascular development (Checchin et al., 2006;

366 Fantin et al., 2010; Rymo et al., 2011), however, the role for microglia in astrocyte

367 template assembly or pruning was not clearly known. In this study, the identification of

368 close microglial interactions with astrocytes in the avascular central and peripheral

369 retina at P0 suggested microglia have a role in facilitating astrocyte template assembly.

370 It is known that astrocytes use laminin basement membrane molecules present in the

371 inner limiting membrane (ILM) as a substrate for migration and template formation

372 (Edwards et al., 2010; Gnanaguru et al., 2013; Tao and Zhang, 2016). Deletion of

373 laminin isoforms or the laminin-binding proteoglycans severely disrupt ILM formation,

374 astrocyte template assembly, and succeeding vascularization (Edwards et al., 2010;

375 Gnanaguru et al., 2013; Tao and Zhang, 2016). In addition to known extracellular matrix

376 cues, our study discovered that the depletion of microglia during the early stages of the

377 astrocyte template assembly phase (from birth), results in abnormal clustering of

378 astrocytes leading to defective template formation. This finding strongly suggests that

379 the spaced honeycomb shaped astrocyte patterning is regulated at multiple cellular and

380 extracellular levels (Fruttiger et al., 1996; Gnanaguru et al., 2013; Tao and Zhang, 2016;

381 Uemura et al., 2006), allowing for the formation of an organized vascular network in the

382 retina during development. 
384 Spatial restructuring during development is an important process that helps retinal

385 maturation and function (Anderson et al., 2019; Chan-Ling et al., 2009; Fuerst et al.,

386 2008; Hughes and Chang-Ling, 2000; Li et al., 2015). It is well documented that during

387 the developmental restructuring process, large numbers of retinal ganglion cells are

388 eliminated (Anderson et al., 2019; Li et al., 2015) and microglia, via C3 dependent

389 signaling mechanisms, phagocytose dying ganglion cells to aid spatial organization

390 (Anderson et al., 2019). Similar to ganglion cells (Anderson et al., 2019; Li et al., 2015),

391 large numbers of astrocytes also undergo cell death peaking at P5 and are removed by

392 microglia through unknown mechanisms (Chan-Ling et al., 2009; Punal et al., 2019).

393 Interestingly, the C3b binding receptor CR3 (also known as CD11b or integrin alpha M)

394 involved in neural pruning, was not associated with astrocyte elimination during retinal

395 vascular development (Punal et al., 2019; Schafer et al., 2012).

397 Several studies have indicated that the C3a anaphylatoxin, and its cognate receptor

398 C3aR involved in immune cell recruitment, mediate astrocyte/microglial crosstalk in the

399 context of neuroinflammation (Lian et al., 2016; Lian et al., 2015; Litvinchuk et al., 2018;

400 Surugiu et al., 2019), thus, in this study we investigated whether C3a/C3aR axis

401 activation also assists in the developmental removal of dying astrocyte bodies/debris by

402 microglia. In contrast to the astrocytic production of C3 in diseased states (Lian et al.,

403 2016; Litvinchuk et al., 2018), our study identified that local expression of C3 and its

404 receptor C3aR were predominantly expressed by microglia during vascular

405 development. It is likely that microglia that are closely interacting with astrocytes deposit 
406 C3 over the template for pruning. Supporting our notion, loss of C3 or C3aR

407 dramatically reduced microglial phagocytic ability to eliminate astrocytes during retinal

408 development. Although we cannot rule out the possible involvement of other

409 complement cleavage fragment receptors, our data provides a compelling role for the

$410 \mathrm{C} 3 a / \mathrm{C} 3 a \mathrm{R}$ signaling axis in astrocyte template pruning.

412 Proper spatial assembly of the astrocytic template is a prerequisite for endothelial tip

413 cell migration for the development of retinal vasculature (Edwards et al., 2010;

414 Gnanaguru et al., 2013; Tao and Zhang, 2016). In light of the previous findings

415 (Hellstrom et al., 2007; Pitulescu et al., 2017), our investigation reveals a plausible role

416 for complement signaling creating a spatially organized astrocytic network to fine tune

417 endothelial tip cell morphogenesis during development.

419 In conclusion, our study identified a novel functional role for the microglia derived C3

420 signaling axis in defining astrocyte spatial pattering and subsequent vascular growth

421 during development.

\section{Materials and Methods}

Mice

427 All animal procedures were performed in accordance with the Massachusetts Eye and 428 Ear Animal Care Committee. Mice were maintained in a room with a 12 hours light/12 
429 hours dark cycle. C57BL6/J (stock \# 00664), C3 KO (stock \# 029661), and C3aR KO

430 (stock \# 033904) breeding pairs were purchased from Jackson Laboratories. C3 KO

431 and C3aR KO mice were crossed to C57BL6/J mice to create a heterozygous line. Pups

432 generated from the heterozygous lines carrying C3+/+ (wild type (WT)), C3-/- (C3 KO),

$433 \mathrm{C} 3 a \mathrm{R}+/+$, and C3aR-/- genotypes were used for the studies. Floxed C3 IRES-tdTomato

434 reporter mice (Kolev et al., 2020) obtained from Dr. Kemper were bred to C57BL6/J

435 mice and maintained on C57BL6/J background. Floxed C3aR-tdTomato reporter knock-

436 in mice (Quell et al., 2017) obtained from Dr. Köhl were maintained on C57BL6/J

437 background.

439 Microglial depletion during development

440 Timed-pregnant mice were maintained on control chow diet or chow diet containing

441 Csfr1 inhibitor (PLX5622, Plexxikon Inc. Berkeley, CA) from gestational day 13.5/14.5.

442 Retinas were collected from pups at postnatal days (P) 1, P5, and P15 for downstream

443 analysis.

445 Isolation and culture of retinal microglia and astrocytes

446 Astrocytes and microglial cells were isolated by immunopanning methodology (Collins

447 and Bohlen, 2018; Zhang et al., 2016). In brief, six-well culture plates were coated with

448 respective secondary antibodies $(10 \mu \mathrm{g} / \mathrm{ml}$ diluted in sterile $50 \mathrm{mM}$ Tris- $\mathrm{HCl}, \mathrm{pH} 9.5)$ by

449 incubating for 1 hour at $37^{\circ} \mathrm{C}$. After washes, culture plates were coated with $2.5 \mu \mathrm{g} / \mathrm{ml}$ of

450 anti-CD11b or anti-integrin beta5 for overnight in the cell culture hood and washed in $1 \mathrm{X}$

451 PBS. P5 retinas ( $n=8)$ were isolated in ice-cold sterile 1X-DPBS (Thermo Fischer 
452 Scientific, Foster City, CA) and incubated in DMEM/F12 containing 5\% fetal bovine

453 serum (FBS), collagenase $\mathrm{D}\left(1 \mathrm{mg} / \mathrm{ml}\right.$, ) and DNase $(0.1 \mathrm{mg} / \mathrm{ml})$ for $30 \mathrm{~min}$ at $37^{\circ} \mathrm{C}$. The

454 retinas were then gently triturated in DMEM/F12 supplemented with $10 \%$ FBS and

455 filtered through a 40 4 m cell strainer to remove cell clumps and debris. The resultant

456 single cell suspension was incubated in secondary antibody only coated well for 10 min

457 at $37^{\circ} \mathrm{C}$ to deplete non-specific cell binding. The unbound cell suspension was then

458 transferred to anti-CD11b coated wells and incubated for $20 \min 37^{\circ} \mathrm{C}$ to capture

459 microglia (culture plate was shaken every 5 min to dislodge non-specific cell binding).

460 After depleting microglia, the cell suspension was transferred to integrin beta 5 coated

461 wells to capture astrocytes and incubated for $45 \mathrm{~min}$ at $37^{\circ} \mathrm{C}$ (culture plates were

462 shaken every 10 min to dislodge non-specific cell binding). After removing the unbound

463 cells, microglia and astrocyte cell culture plates were thoroughly washed in serum free

464 DMEM/F12. Microglial cells were grown and maintained in DMEM/F12 supplemented

465 with $5 \%$ FBS and $10 \mathrm{ng} / \mathrm{ml}$ of recombinant mouse csf1, and astrocytes were grown and

466 maintained in DMEM/F12 supplemented with $10 \% \mathrm{FBS}$ and $\mathrm{N}-2$.

In vitro phagocytosis assay

469 To examine microglial phagocytosis of dead astrocyte bodies, $\sim 2,500$ microglial cells

470 were plated on a $12 \mathrm{~mm}$ glass coverslip and maintained for 48 hours. Astrocyte cell

471 suspensions were labeled with Vybrant ${ }^{\mathrm{TM}}$ Dil cell labeling solution as suggested by the

472 manufacturer (Thermo Fischer Scientific, Foster City, CA) and cell death was induced

473 by incubating the labeled astrocyte cell suspension with $1 \mu \mathrm{M}$ staurosporine (Cayman

474 Chemical Company, Ann Arbor, MI). Microglial cells were then treated with Dil-labeled 
475 dead astrocyte bodies in 1:1 ratio and incubated for 2 hours. For receptor neutralization 476 assays, microglial cells were preincubated with $20 \mu \mathrm{g} / \mathrm{ml}$ of anti-C3aR antibody (Catalog

477 \# MAB10417) or control IgG (R\&D Systems, Inc. Minneapolis, MN) suspended in

478 DMEM/F12 supplemented with 5\% heat-inactivated FBS, followed by treatment with Dil-

479 labeled astrocyte dead bodies in the presence of antibodies for $2 \mathrm{hr}$ at $37^{\circ} \mathrm{C}$. After

480 thorough washes, cells were fixed in 4\% PFA, blocked for $1 \mathrm{hr}$ at room temperature (in

$48110 \%$ fetal bovine serum, $0.05 \%$ triton-X100, and $0.01 \%$ sodium azide in $1 \mathrm{X}$ PBS),

482 followed by incubation with P2ry12 antibody (Catalog\# AS-55043A, AnaSpec, Fremont, $483 \mathrm{CA}$ ) overnight at $4^{\circ} \mathrm{C}$ and then incubated with goat anti-rabbit Alexa Fluor 488 or 647

484 (Thermo Fischer Scientific, Foster City, CA), images were acquired using epifluroscent 485 microscope (Axio Observer Zeiss).

487 Flatmount preparation and immunostaining

488 Retinal flatmounts were prepared as described previously (Gnanaguru et al., 2013). In

489 brief, eyes were fixed in 2\% paraformaldehyde (PFA) for 10-15 min and then dissected 490 in 1X PBS. After discarding the anterior chamber and lens, the retina was separated

491 from the sclera. Retinal flatmounts were prepared with four radial incisions and the

492 flatmounts were then stored in methanol at $-20^{\circ} \mathrm{C}$ until immunostaining was performed.

493 For immunohistochemistry, retinas were rehydrated in 1 X PBS and washed at $3 \mathrm{X}$ and

494 blocked in blocking buffer (10\% fetal bovine serum, 0.05\%triton-X100, and 0.01\%

495 sodium azide in $1 \mathrm{X}$ PBS) for $2 \mathrm{hr}$ at room temperature. Following blocking, retinas were

496 then incubated with primary antibodies for $24 \mathrm{hr}$ at $4^{\circ} \mathrm{C}$. After washes in $1 \mathrm{X}$ PBS, retinal

497 flatmounts were incubated with respective secondary antibodies for $4 \mathrm{hr}$ at room 
498 temperature or overnight at $4^{\circ} \mathrm{C}$. The flatmounts were then washed in $1 \mathrm{X}$ PBS and

499 mounted onto a slide using anti-fade medium (Permaflour, Thermo Fisher Scientific,

500 Waltham, MA).

501 Primary antibodies used: Rat anti-Pdgfra (CD140A, clone: APA5, BD Biosciences, San

502 Jose, CA), anti-chicken GFAP (Aves Labs, Inc, Davis, CA), rabbit anti-P2ry12 (a gift

503 from Dr.Butovsky, Brigham and Women's Hospital), rat anti-mouse CD68 (Clone: FA-

504 11, Biolegend, Dedham, MA), anti-rabbit Iba1(Catalog\# 019-19741, FUJIFILM Wako

505 Chemicals, Richmond, VA), and goat anti-CD31 (Catalog \# AF3628, R\&D Systems,

506 Inc. Minneapolis, MN).

507 Secondary antibodies used: Donkey anti-chicken 594 (Biotium, Inc. Fremont, CA), anti-

508 rat-594, donkey anti-rabbit-488, and donkey anti-goat-647 (Thermo Fisher Scientific,

509 Waltham, MA)

510

511 Cryosectioning

512 The enucleated eyes were fixed in 4\% PFA for $2 \mathrm{hr}$ at room temperature and then the

513 dissected posterior eyecups were cryopreserved in $10 \%, 20 \%$, and $30 \%$ sucrose and

514 then frozen in Tissue-Tek® O.C.T compound (Ted Pella, Inc. Redding, CA). The

515 eyecups were then cut at $12 \mu \mathrm{m}$ thickness and used for immunohistochemistry.

\section{Flow cytometry}

518 Singe cell suspensions were prepared from retinas that were dissected in ice-cold

519 HBSS and then incubated with collagenase D $(1 \mathrm{mg} / \mathrm{ml})$ and DNAse $(0.1 \mathrm{mg} / \mathrm{ml})$

520 (Millipore Sigma) in DMEM/F12 supplemented with 5\% FBS for 30 min at $37^{\circ} \mathrm{C}$. After 
521 filtering the cells through a $40 \mu \mathrm{m}$ cell strainer, single cell suspensions were blocked with

522 anti-mouse CD16/32 monoclonal antibody and then stained with APC anti-Pdgfra

523 (CD140a, clone: APA5), and PE anti-p2ry12 (clone: S16007D, Biolegend, Dedham,

524 MA). Dead cells were distinguished with DAPI stain. The data for flow cytometry was

525 acquired by Cytoflex S (Beckman Coulter, Indianapolis, Indiana) and analyzed using

526 FlowJo version 10.1.

527

\section{Real-time PCR}

529 Retinas were isolated in ice-cold PBS and RNA was extracted using RNA STAT-60 ${ }^{\text {TM }}$

530 as recommended by the manufacturer. After determining the RNA concentration using

531 NanoDrop ${ }^{\mathrm{TM}}$, 500ng of total RNA was used for cDNA synthesis using SuperScript ${ }^{\mathrm{TM}}$ IV

532 VILO ${ }^{\text {TM }}$ Master Mix. Real-time PCR reactions were performed in the CFX384 ${ }^{\text {TM }}$ Real-

533 time PCR platform (Bio-Rad, Hercules, CA) using SYBR Green master mix (Applied

534 biosystems, Thermo Fischer Scientific, Foster City, CA) to determine the relative

535 expression level of Pdgfa, P2ry12, Tmem119, Csf1r, Vegfc, Vegf120, Vegf164, and

536 Vegf188 (Darland et al., 2011). Sequences of primers used: Ppia (TTC ACC TTC CCA

537 AAG ACC AC and CAA ACA CAA ACG GTT CCC AG), Pdgfa

538 (ATTAACCATGTGCCCGAGAA and GTATCTCGTAAATGACCGTCCTG), P2ry12

539 (CCAGTCTGCAAGTTCCACTAAC and GAGAAGGTGGTATTGGCTGAG, Tmem119

540 (GGTCCTTCACCCAGAGC and GGAGTGACACAGAGTAGGC), Csf1r (TGT

541 ATGTCTGTCATGTCTCTGC and AGGTGTAGCTATTGCCTTCG), Vegfc

542 (GAAGTTCCACCATCAAACATGC and CAGCGGCAT ACTTCTTCACTA), Vegf120

543 (GCCAGCACATAGGAGAGATGAGC and CGGCTTGTCACATTTTTCTGG), Vegf164 
(GCCAGCACATAGGAGAGATGAGC and CAAGGCTCACAGTGATTTTCTGG), and

Vegf188 (GCCAGCACATAGGAGAGATGAGC and AACAAGGCTCACAGTGAACGCT).

\section{Image analysis}

548 Samples were imaged using an epifluroscent microscope (Axio Observer Zeiss) or

549 confocal microscopy (SP8, Leica). For 3D image reconstruction of Z-stack images,

550 Amira 2019.4 software tool was used.

\section{Analysis of astrocyte and vascular coverage}

552 Tiled images of the entire retinal surface immunostained for Pdgfra or CD31 were

553 acquired. Using NIH-ImageJ (version 2.1.0/1.55c)) software, scale measurements were

554 set and an outline of the astrocyte or vascular growth front was created using manual

555 freehand selection tool total area of the Pdgfra or CD31 immunostained area was

556 measured. Tip cells were manually quantified in all four quadrants using ImageJ multi-

557 point tool.

558 Microglia immunostained cellular area measurement

559 Images of retinal flatmounts co-labeled with CD31 and P2ry12 or CD31 and Iba1 were

560 acquired in all the retinal quadrants near the tip cell and avascular peripheral regions by

561 confocal microscopy. Outline of P2ry12 or lba1 stained cell area was manually drawn

562 using Image $\mathrm{J}$ (version 2.1.0/1.53c) freehand selection tool and each immunostained

563 cell area was measured and quantified.

564 Analysis of astrocyte and vessel density, branching, and lacunarity

565 Astrocyte and blood vessel covered area were quantified using ImageJ (version

$5662.1 .0 / 1.53 \mathrm{c})$ Angiogenesis Analyzer plugin tool. In brief, using free hand selection tool 
567 the edges of astrocyte or blood vessel stained region was manually selected and the

568 area was determined. Tip cells were manually quantified in all four quadrants using

569 ImageJ Wand tracing tool. Vascular density, branching index, and lacunacity was

570 analyzed using ImageJ Angiogenesis Analyzer.

571

\section{Statistical analysis}

573 All the other graphs and statistical analysis were performed using Prism version 9

574 software. All data were presented as the mean +/- SEM. Statistical differences between

575 the two groups were determined by unpaired t test. The 'n' and the level of statistical

576 significance were noted in the legend of each figure.

577

578 Data availability

579 Images and flow cytometry data used for the analysis are uploaded to Harvard

580 institutional repository. Upon acceptance these data will be publicly available

581 (https://dataverse.harvard.edu/dataset.xhtml?persistentld=doi:10.7910/DVN/CDJYDT).

582 All raw data will also be available by reaching out to the Lead Contact, Gopalan

583 Gnanaguru (gopalan_gnanaguru@meei.harvard.edu).

584

\section{Author contributions}

586 GG and KMC designed research and wrote the manuscript; GG performed research

587 and analyzed data; SJT and KY analyzed data; RM performed embryonic study, CK and 588 JK generated and provided reporter mice. 


\section{Acknowledgments}

591 This work was supported by NIH/National Eye Institute Grant R01EY032502 (to G.G).

592 We thank Plexxikon, inc, for providing PLX5622 chow diet. We sincerely thank

593 Dr.Butovsky (Department of Neurology, Brigham and Women's Hospital, Harvard

594 Medical School) for providing the P2ry12 antibody and Dr. Claudia Kemper

595 (Immunology Center, National Heart, Lung, and Blood Institute, NIH, Bethesda, MD,

USA 20892) for providing the Floxed C3 IRES-tdTomato reporter mice.

\section{Conflict of interest}

The authors declare no competing interests

600

\section{References:}

Abbott, N.J., Ronnback, L., and Hansson, E. (2006). Astrocyte-endothelial interactions at the blood-brain barrier. Nat Rev Neurosci 7, 41-53. and Vetter, M.L. (2019). Complement Targets Newborn Retinal Ganglion Cells for Phagocytic Elimination by Microglia. J Neurosci 39, 2025-2040. apoptotic cells resulting in enhanced $\mathrm{T}$-cell responses to cargo-derived antigens. Proc Natl Acad Sci U S A 111, 1503-1508.

Benard, M., Raoult, E., Vaudry, D., Leprince, J., Falluel-Morel, A., Gonzalez, B.J., Galas, L., Vaudry, H., and Fontaine, M. (2008). Role of complement anaphylatoxin receptors $(\mathrm{C} 3 \mathrm{aR}, \mathrm{C} 5 \mathrm{aR})$ in the development of the rat cerebellum. Mol Immunol 45, 3767-3774.

Butovsky, O., Jedrychowski, M.P., Moore, C.S., Cialic, R., Lanser, A.J., Gabriely, G., Koeglsperger, T., Dake, B., Wu, P.M., Doykan, C.E., et al. (2014). Identification of a unique TGF-beta-dependent molecular and functional signature in microglia. Nat

618 Neurosci 17, 131-143. characterization of astrocyte precursor cells (APCs) and astrocytes in developing rat retinae: differentiation, proliferation, and apoptosis. Glia 57, 39-53. 
622 Checchin, D., Sennlaub, F., Levavasseur, E., Leduc, M., and Chemtob, S. (2006).

623 Potential role of microglia in retinal blood vessel formation. Invest Ophthalmol Vis Sci

624 47, 3595-3602.

625 Chitu, V., and Stanley, E.R. (2017). Regulation of Embryonic and Postnatal

626 Development by the CSF-1 Receptor. Curr Top Dev Biol 123, 229-275.

627 Collins, H.Y., and Bohlen, C.J. (2018). Isolation and Culture of Rodent Microglia to

628 Promote a Dynamic Ramified Morphology in Serum-free Medium. J Vis Exp.

629 Cong, Q., Soteros, B.M., Wollet, M., Kim, J.H., and Sia, G.M. (2020). The endogenous

630 neuronal complement inhibitor SRPX2 protects against complement-mediated synapse

631 elimination during development. Nat Neurosci 23, 1067-1078.

632 Coulthard, L.G., Hawksworth, O.A., Conroy, J., Lee, J.D., and Woodruff, T.M. (2018).

633 Complement C3a receptor modulates embryonic neural progenitor cell proliferation and

634

635 cognitive performance. Mol Immunol 101, 176-181.

Darland, D.C., Cain, J.T., Berosik, M.A., Saint-Geniez, M., Odens, P.W., Schaubhut, G.J., Frisch, S., Stemmer-Rachamimov, A., Darland, T., and D'Amore, P.A. (2011). Vascular endothelial growth factor (VEGF) isoform regulation of early forebrain development. Dev Biol 358, 9-22.

Dorrell, M.I., Aguilar, E., and Friedlander, M. (2002). Retinal vascular development is

640 mediated by endothelial filopodia, a preexisting astrocytic template and specific Rcadherin adhesion. Invest Ophthalmol Vis Sci 43, 3500-3510.

642 Edwards, M.M., Mammadova-Bach, E., Alpy, F., Klein, A., Hicks, W.L., Roux, M., Simon-Assmann, P., Smith, R.S., Orend, G., Wu, J., et al. (2010). Mutations in Lama1 disrupt retinal vascular development and inner limiting membrane formation. J Biol Chem 285, 7697-7711. Elmore, M.R., Najafi, A.R., Koike, M.A., Dagher, N.N., Spangenberg, E.E., Rice, R.A., Kitazawa, M., Matusow, B., Nguyen, H., West, B.L., et al. (2014). Colony-stimulating factor 1 receptor signaling is necessary for microglia viability, unmasking a microglia progenitor cell in the adult brain. Neuron 82, 380-397. Wilson, S.W., and Ruhrberg, C. (2010). Tissue macrophages act as cellular chaperones for vascular anastomosis downstream of VEGF-mediated endothelial tip cell induction. Blood 116, 829-840.

Fruttiger, M. (2007). Development of the retinal vasculature. Angiogenesis 10, 77-88. Fruttiger, M., Calver, A.R., Kruger, W.H., Mudhar, H.S., Michalovich, D., Takakura, N., Nishikawa, S., and Richardson, W.D. (1996). PDGF mediates a neuron-astrocyte interaction in the developing retina. Neuron 17, 1117-1131.

658 Fruttiger, M., Calver, A.R., and Richardson, W.D. (2000). Platelet-derived growth factor

659 is constitutively secreted from neuronal cell bodies but not from axons. Curr Biol 10, 660 1283-1286.

661 Fuerst, P.G., Koizumi, A., Masland, R.H., and Burgess, R.W. (2008). Neurite

662

663 arborization and mosaic spacing in the mouse retina require DSCAM. Nature 451, 470474. 
667 Gnanaguru, G., Bachay, G., Biswas, S., Pinzon-Duarte, G., Hunter, D.D., and Brunken, 668 W.J. (2013). Laminins containing the beta2 and gamma3 chains regulate astrocyte migration and angiogenesis in the retina. Development 140, 2050-2060.

670 Grajales-Esquivel, E., Luz-Madrigal, A., Bierly, J., Haynes, T., Reis, E.S., Han, Z., 671 Gutierrez, C., McKinney, Z., Tzekou, A., Lambris, J.D., et al. (2017). Complement 672 component $\mathrm{C} 3 \mathrm{aR}$ constitutes a novel regulator for chick eye morphogenesis. Dev Biol 673 428, 88-100.

674 Hartmann, K., Henz, B.M., Kruger-Krasagakes, S., Kohl, J., Burger, R., Guhl, S., Haase, 675 I., Lippert, U., and Zuberbier, T. (1997). C3a and C5a stimulate chemotaxis of human 676 mast cells. Blood 89, 2863-2870.

677 Hartnett, M.E. (2020). Retinopathy of Prematurity: Evolving Treatment With Anti678 Vascular Endothelial Growth Factor. Am J Ophthalmol 218, 208-213.

679 Hellstrom, M., Phng, L.K., Hofmann, J.J., Wallgard, E., Coultas, L., Lindblom, P., Alva, 680 J., Nilsson, A.K., Karlsson, L., Gaiano, N., et al. (2007). Dll4 signalling through Notch1 681 regulates formation of tip cells during angiogenesis. Nature 445, 776-780.

682 Hilla, A.M., Diekmann, H., and Fischer, D. (2017). Microglia Are Irrelevant for Neuronal Degeneration and Axon Regeneration after Acute Injury. J Neurosci 37, 6113-6124. Holers, V.M. (2014). Complement and its receptors: new insights into human disease. Annu Rev Immunol 32, 433-459.

686 Holmstrom, G., van Wijngaarden, P., Coster, D.J., and Williams, K.A. (2007). Genetic susceptibility to retinopathy of prematurity: the evidence from clinical and experimental animal studies. Br J Ophthalmol 91, 1704-1708. Hughes, S., and Chang-Ling, T. (2000). Roles of endothelial cell migration and apoptosis in vascular remodeling during development of the central nervous system.

691 Microcirculation 7, 317-333.

692 Inafuku, S., Klokman, G., and Connor, K.M. (2018). The Alternative Complement System Mediates Cell Death in Retinal Ischemia Reperfusion Injury. Front Mol Neurosci $11,278$. expression of $\mathrm{C} 3 \mathrm{a}$ and $\mathrm{C} 5 \mathrm{a}$ exacerbate demyelination severity in the cuprizone model. Mol Immunol 48, 219-230. Kim, C., Smith, K.E., Castillejos, A., Diaz-Aguilar, D., Saint-Geniez, M., and Connor, K.M. (2016). The alternative complement pathway aids in vascular regression during the early stages of a murine model of proliferative retinopathy. FASEB J 30, 1300-1305. Kolev, M., West, E.E., Kunz, N., Chauss, D., Moseman, E.A., Rahman, J., Freiwald, T., Balmer, M.L., Lotscher, J., Dimeloe, S., et al. (2020). Diapedesis-Induced Integrin Signaling via LFA-1 Facilitates Tissue Immunity by Inducing Intrinsic Complement C3 Expression in Immune Cells. Immunity 52, 513-527 e518.

Langen, U.H., Ayloo, S., and Gu, C. (2019). Development and Cell Biology of the BloodBrain Barrier. Annu Rev Cell Dev Biol 35, 591-613.

707 Li, S., Sukeena, J.M., Simmons, A.B., Hansen, E.J., Nuhn, R.E., Samuels, I.S., and 708 Fuerst, P.G. (2015). DSCAM promotes refinement in the mouse retina through cell death and restriction of exploring dendrites. J Neurosci 35, 5640-5654. Lian, H., Litvinchuk, A., Chiang, A.C., Aithmitti, N., Jankowsky, J.L., and Zheng, H. (2016). Astrocyte-Microglia Cross Talk through Complement Activation Modulates Amyloid Pathology in Mouse Models of Alzheimer's Disease. J Neurosci 36, 577-589. 
713 Lian, H., Yang, L., Cole, A., Sun, L., Chiang, A.C., Fowler, S.W., Shim, D.J., Rodriguez-

714 Rivera, J., Taglialatela, G., Jankowsky, J.L., et al. (2015). NFkappaB-activated astroglial release of complement $\mathrm{C} 3$ compromises neuronal morphology and function associated with Alzheimer's disease. Neuron 85, 101-115. Litvinchuk, A., Wan, Y.W., Swartzlander, D.B., Chen, F., Cole, A., Propson, N.E., Wang, Q., Zhang, B., Liu, Z., and Zheng, H. (2018). Complement C3aR Inactivation Attenuates Tau Pathology and Reverses an Immune Network Deregulated in Tauopathy Models and Alzheimer's Disease. Neuron 100, 1337-1353 e1335. Merle, N.S., Church, S.E., Fremeaux-Bacchi, V., and Roumenina, L.T. (2015). Complement System Part I - Molecular Mechanisms of Activation and Regulation. Front Immunol 6, 262.

Mevorach, D., Mascarenhas, J.O., Gershov, D., and Elkon, K.B. (1998). Complementdependent clearance of apoptotic cells by human macrophages. J Exp Med 188, 23132320.

Mukai, R., Okunuki, Y., Husain, D., Kim, C.B., Lambris, J.D., and Connor, K.M. (2018). The Complement System Is Critical in Maintaining Retinal Integrity during Aging. Front Aging Neurosci 10, 15. Okunuki, Y., Mukai, R., Nakao, T., Tabor, S.J., Butovsky, O., Dana, R., Ksander, B.R., and Connor, K.M. (2019). Retinal microglia initiate neuroinflammation in ocular autoimmunity. Proc Natl Acad Sci U S A 116, 9989-9998. Okunuki, Y., Mukai, R., Pearsall, E.A., Klokman, G., Husain, D., Park, D.H., Korobkina, E., Weiner, H.L., Butovsky, O., Ksander, B.R., et al. (2018). Microglia inhibit photoreceptor cell death and regulate immune cell infiltration in response to retinal detachment. Proc Natl Acad Sci U S A 115, E6264-E6273.

Outtz, H.H., Tattersall, I.W., Kofler, N.M., Steinbach, N., and Kitajewski, J. (2011). Notch1 controls macrophage recruitment and Notch signaling is activated at sites of endothelial cell anastomosis during retinal angiogenesis in mice. Blood 118, 3436-3439. Paolicelli, R.C., Bolasco, G., Pagani, F., Maggi, L., Scianni, M., Panzanelli, P., Giustetto, M., Ferreira, T.A., Guiducci, E., Dumas, L., et al. (2011). Synaptic pruning by microglia is necessary for normal brain development. Science 333, 1456-1458.

Pitulescu, M.E., Schmidt, I., Giaimo, B.D., Antoine, T., Berkenfeld, F., Ferrante, F., Park, H., Ehling, M., Biljes, D., Rocha, S.F., et al. (2017). Dll4 and Notch signalling couples sprouting angiogenesis and artery formation. Nat Cell Biol 19, 915-927. Punal, V.M., Paisley, C.E., Brecha, F.S., Lee, M.A., Perelli, R.M., Wang, J., O'Koren, E.G., Ackley, C.R., Saban, D.R., Reese, B.E., et al. (2019). Large-scale death of retinal astrocytes during normal development is non-apoptotic and implemented by microglia. PLoS Biol 17, e3000492.

Quell, K.M., Karsten, C.M., Kordowski, A., Almeida, L.N., Briukhovetska, D., Wiese, A.V., Sun, J., Ender, F., Antoniou, K., Schroder, T., et al. (2017). Monitoring C3aR Expression Using a Floxed tdTomato-C3aR Reporter Knock-in Mouse. J Immunol 199, 688-706.

756

Roy, C., Gupta, A., Fisette, A., Lapointe, M., Poursharifi, P., Richard, D., Lu, H., Lu, B., Gerard, N., Gerard, C., et al. (2013). C5a receptor deficiency alters energy utilization and fat storage. PLoS One 8, e62531. 
757 Rymo, S.F., Gerhardt, H., Wolfhagen Sand, F., Lang, R., Uv, A., and Betsholtz, C.

758 (2011). A two-way communication between microglial cells and angiogenic sprouts

759 regulates angiogenesis in aortic ring cultures. PLoS One 6, e15846.

760 Santos, A.M., Calvente, R., Tassi, M., Carrasco, M.C., Martin-Oliva, D., Marin-Teva, 761 J.L., Navascues, J., and Cuadros, M.A. (2008). Embryonic and postnatal development 762 of microglial cells in the mouse retina. J Comp Neurol 506, 224-239.

763 Schafer, D.P., Lehrman, E.K., Kautzman, A.G., Koyama, R., Mardinly, A.R., Yamasaki, 764 R., Ransohoff, R.M., Greenberg, M.E., Barres, B.A., and Stevens, B. (2012). Microglia sculpt postnatal neural circuits in an activity and complement-dependent manner. Neuron 74, 691-705.

Schnitzer, J. (1988). Astrocytes in the guinea pig, horse, and monkey retina: their occurrence coincides with the presence of blood vessels. Glia 1, 74-89.

770 Stone, J., and Dreher, Z. (1987). Relationship between astrocytes, ganglion cells and vasculature of the retina. J Comp Neurol 255, 35-49. Popa-Wagner, A. (2019). Intracortical Administration of the Complement C3 Receptor Antagonist Trifluoroacetate Modulates Microglia Reaction after Brain Injury. Neural Plast $2019,1071036$.

Sweigard, J.H., Matsumoto, H., Smith, K.E., Kim, L.A., Paschalis, E.I., Okonuki, Y., Castillejos, A., Kataoka, K., Hasegawa, E., Yanai, R., et al. (2015). Inhibition of the alternative complement pathway preserves photoreceptors after retinal injury. Sci Transl Med 7, 297ra116. Basement Membrane Assembly to Control Astrocyte Migration and Angiogenesis. Cell Rep 17, 1832-1844. as a proangiogenic switch by regulating extracellular assembly of fibronectin matrices in retinal astrocytes. J Clin Invest 116, 369-377.

Valdearcos, M., Robblee, M.M., Benjamin, D.I., Nomura, D.K., Xu, A.W., and Koliwad, S.K. (2014). Microglia dictate the impact of saturated fat consumption on hypothalamic inflammation and neuronal function. Cell Rep 9, 2124-2138. Watanabe, T., and Raff, M.C. (1988). Retinal astrocytes are immigrants from the optic nerve. Nature 332, 834-837.

790 Wong, T.Y., Cheung, C.M., Larsen, M., Sharma, S., and Simo, R. (2016). Diabetic retinopathy. Nat Rev Dis Primers 2, 16012.

792 Zhang, Y., Sloan, S.A., Clarke, L.E., Caneda, C., Plaza, C.A., Blumenthal, P.D., Vogel, H., Steinberg, G.K., Edwards, M.S., Li, G., et al. (2016). Purification and Characterization of Progenitor and Mature Human Astrocytes Reveals Transcriptional and Functional Differences with Mouse. Neuron 89, 37-53.

Zhang, Y., and Stone, J. (1997). Role of astrocytes in the control of developing retinal vessels. Invest Ophthalmol Vis Sci 38, 1653-1666. 
802

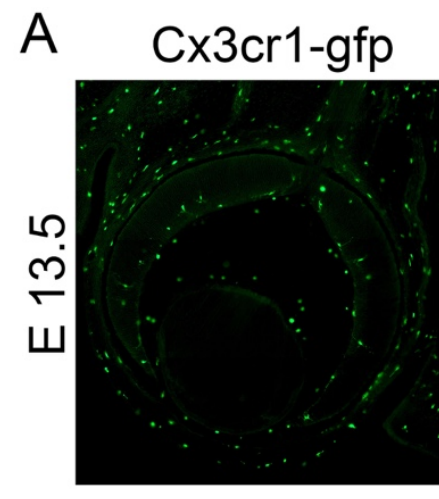

B

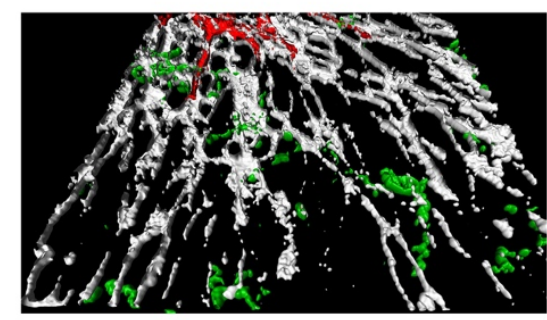

Near $\mathrm{ONH}$
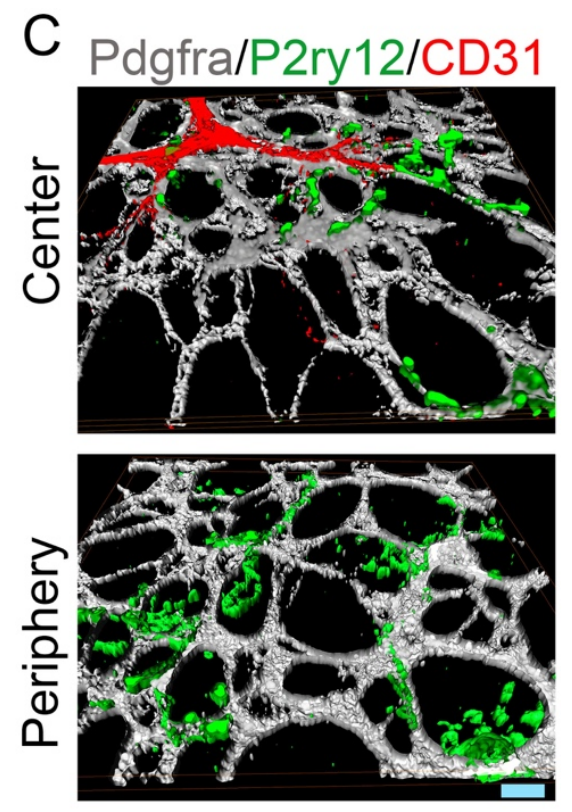

803

804

P2ry12
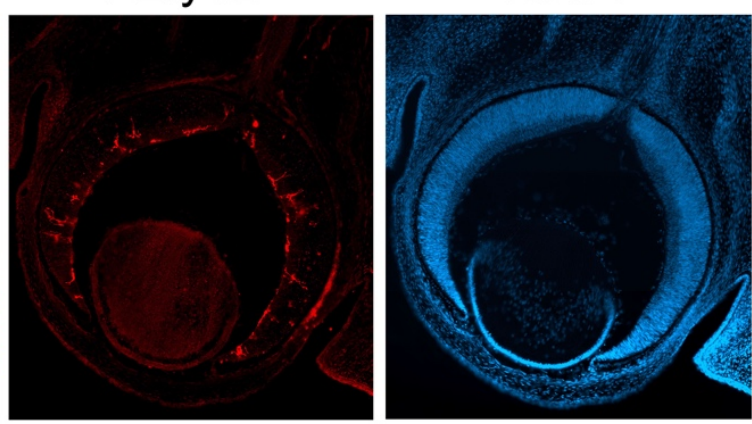

Pdgfra/P2ry12/CD31

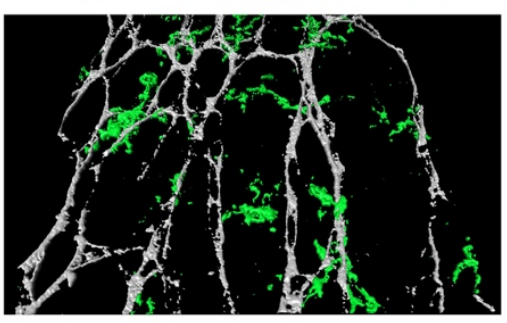

Center

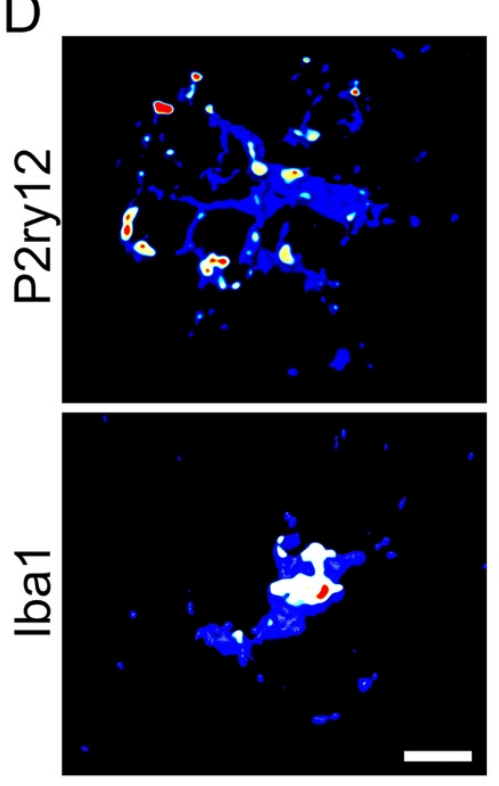

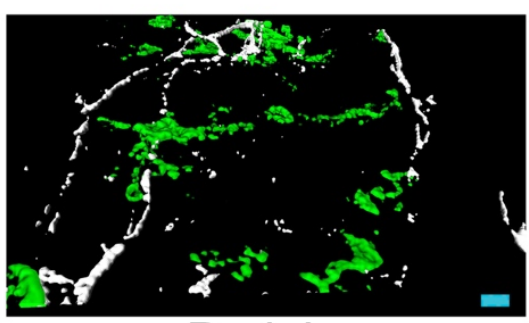

Periphery

$E$

Proximal to tip cells
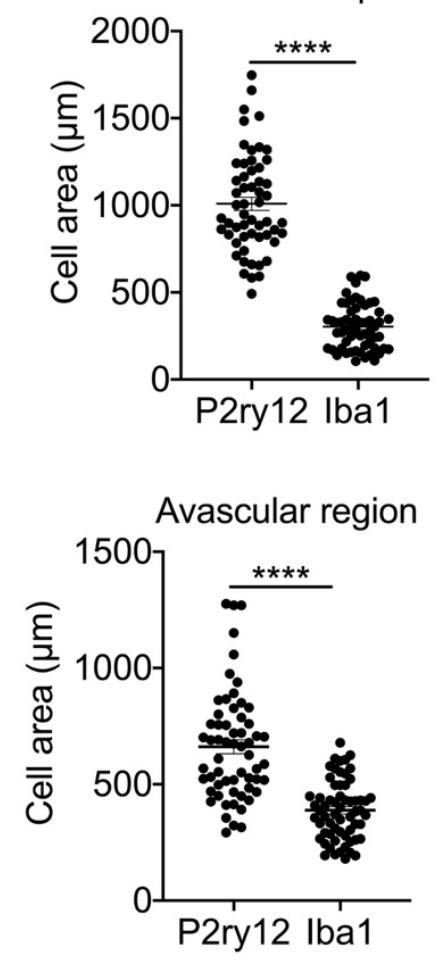
805 Figure 1. Microglia populate the retina early in embryonic development and are recruited

806 to sites of astrocyte template formation and vascularization. A. Cross section of an

807 embryonic (E) day 13.5 Cx3cr1-gfp expressing retina immunostained for P2ry12 and

808 DAPI nuclear stain (blue). Overlaid images reveal Cx3cr1-gfp and P2ry12 colocalization

809 within a microglia cell population. (B and C) Retinal flatmounts prepared from P0 and P5

810 pups were immunostained for Pdgfra, P2ry12, and CD31 and Z-stack images were

811 acquired and 3D-reconstructed to examine microglial interaction with astrocytes and

812 endothelial tip cells early during vascular development. B. Representative 3D-

813 reconstructed image near the optic nerve head $(\mathrm{ONH})$, central, and peripheral retina

814 revealing microglia (P2ry12-green) have a close association with astrocytes (Pdgfra-

815 grey), and endothelial tip cells (CD31-red) (n=3). C. Representative 3D-reconstructed

816 image of P5 C57BL/6J retinal flatmount immunostained for Pdgfra (astrocyte marker),

817 P2ry12 (microglial marker), and CD31 (endothelial cell marker). Confocal z-stack images

818 were taken near the leading tip cell area and avascular peripheral retina to visualize

819 microglial interaction with endothelial cells and astrocytes $(n=5)$. D. Representative 3D

820 reconstructed P5 retinal flatmount immunostained for either P2ry12 or lba1 revealing

821 microglia morphology. Areas with increased intensity are indicated as hotspots $(n=4)$. E.

822 Quantification of P2ry12 and Iba1 localized expression around tip cells and avascular

823 regions of the retina $(n=4)$. Scale bars: $A$ is $100 \mu \mathrm{m}, \mathrm{B}, \mathrm{C}$, and $\mathrm{D}$ are $10 \mu \mathrm{m}$. All error bars

824 represent \pm S.E.M. Statistical differences were calculated by unpaired $t$ test. ${ }^{* * *} \mathrm{P}<$

$825 \quad 0.0001$

826

827 


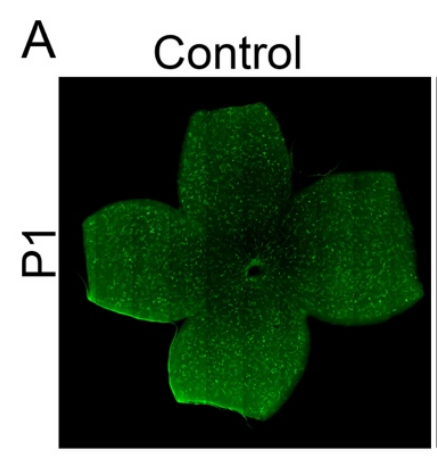

C

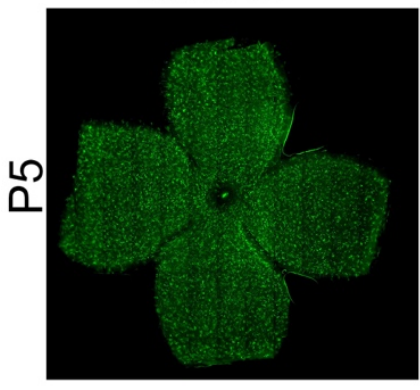

Control
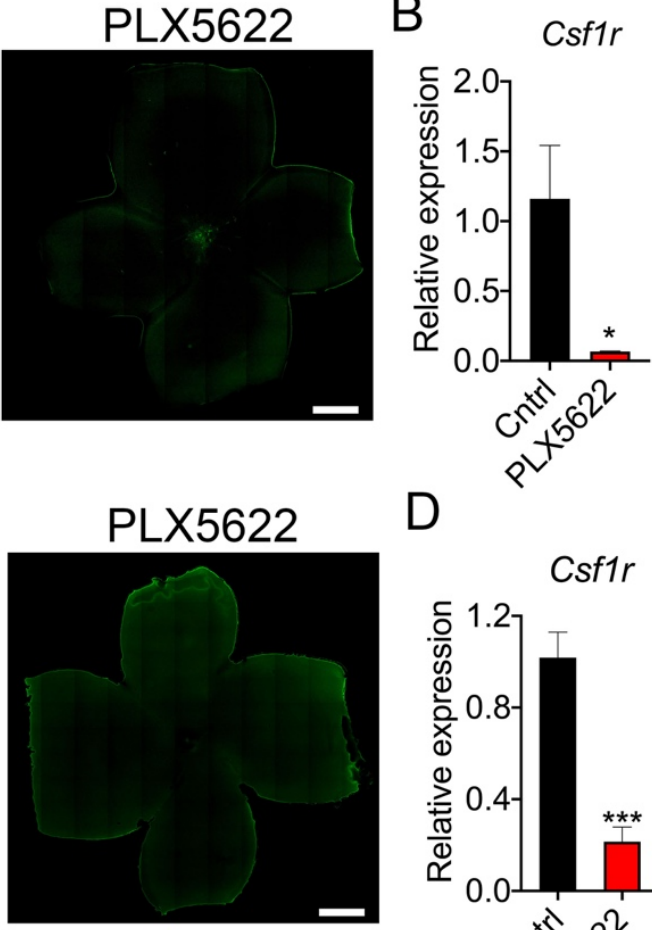

D

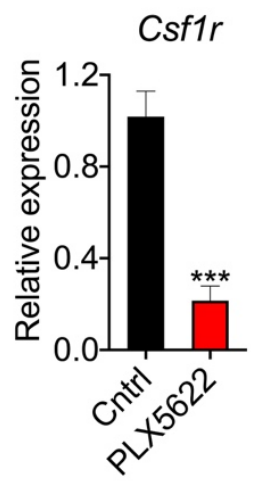

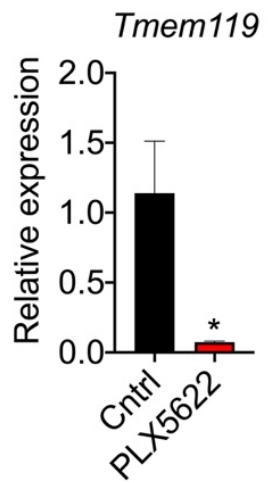
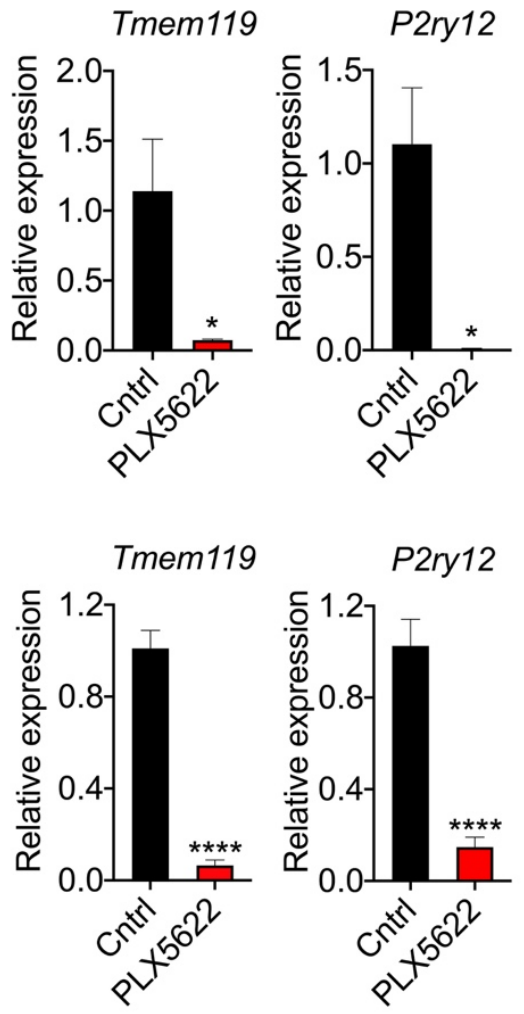
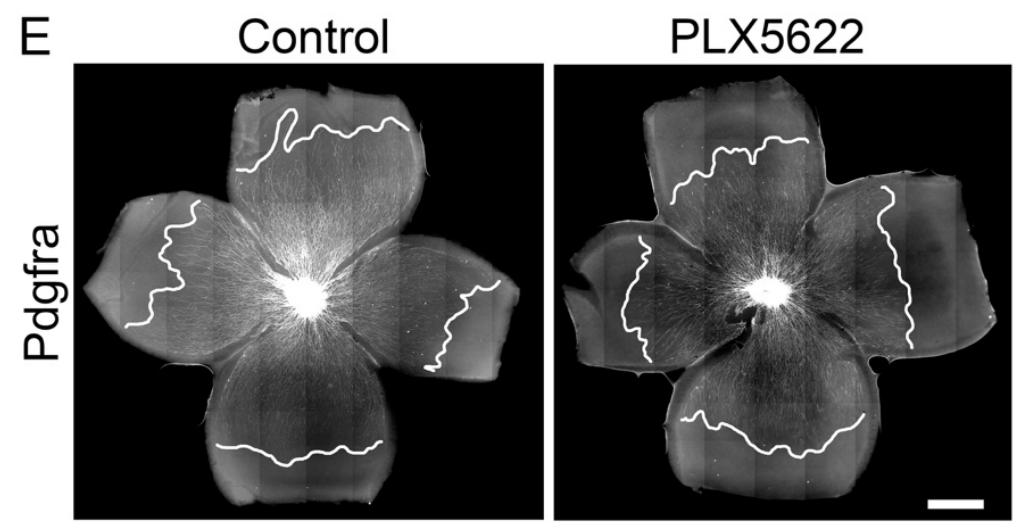

Astrocyte coverage

Figure 2. Csf1r specific antagonist effectively depletes retinal microglia early in

vascular developmental stage. Time-pregnant C57BL/6J mice from gestational day

E13.5/E14.5 were fed a control or Csf1r specific antagonist (PLX5622) diet and

833 littermate controls were analyzed at P1 and P5 developmental time points. (A-F). A.

834 Representative P1 retinal flatmounts from control or PLX5622 treated groups showing

835 staining of P2ry12 (Green). B. Real-time PCR analysis of relative gene expression 
836 levels of microglia markers, Csf1r, P2ry12, and Tmem119 at P1 $(n=3)$ in the retinas of

837 control or PLX5622 groups. C. Representative anti-P2ry12 immunostaining of retinal

838 flatmounts showing microglial distribution at P5. D. Real-time PCR analysis of relative

839 gene expression levels of microglia markers, Csf1r, P2ry12, and Tmem119 at P5 (n=5)

840 in the retinas of control or PLX5622 groups. E. P1 retinal flatmounts isolated from

841 control or PLX5622 groups were immunostained for Pdgfra, the percentage of astrocyte

842 coverage (indicated by dotted lines in images) was quantified and shown in the graph

$843(n=4)$. Scale bar: A, C, and D are 500 $\mu$ m. All error bars represent \pm S.E.M. Statistical

844 differences between control and PLX-5622 group was calculated by unpaired $t$ test. * P

$845<0.05,{ }^{* * *} \mathrm{P}<0.001,{ }^{* * *} \mathrm{P}<0.0001$

846

847

848

849

850

851

852

853

854

855

856 

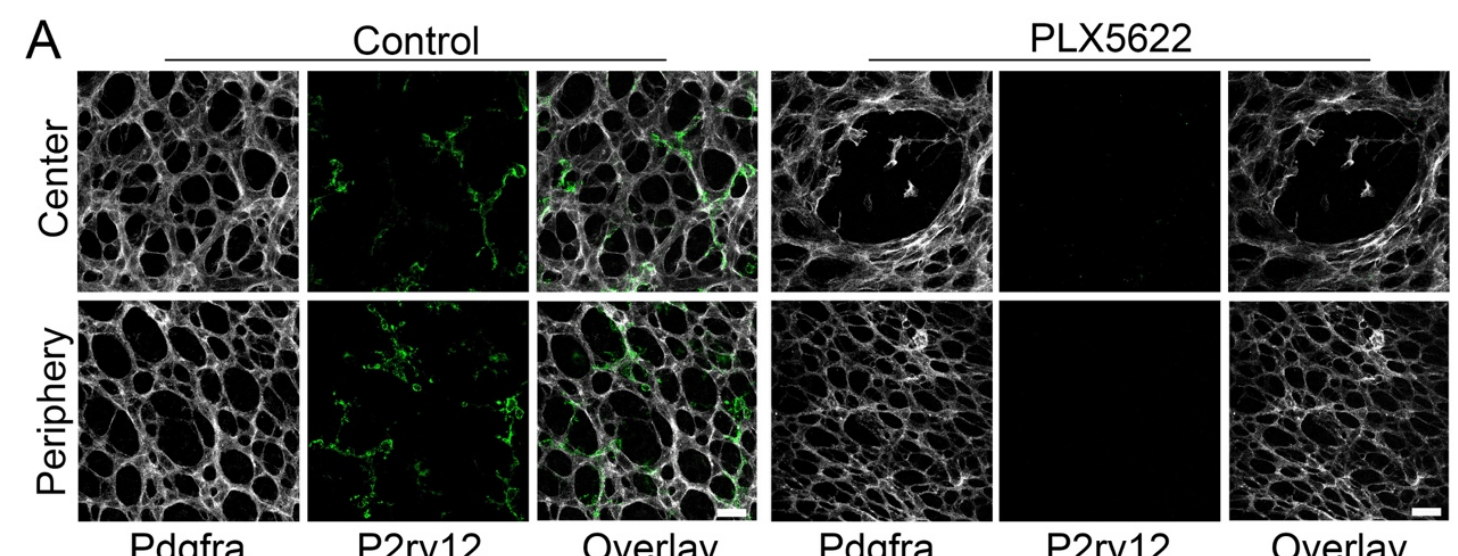

Pdgfra

P2ry12
Pdgfra

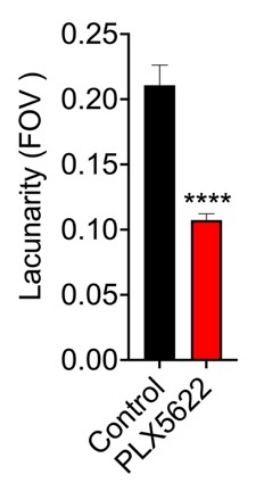

P2ry12

Overlay

B
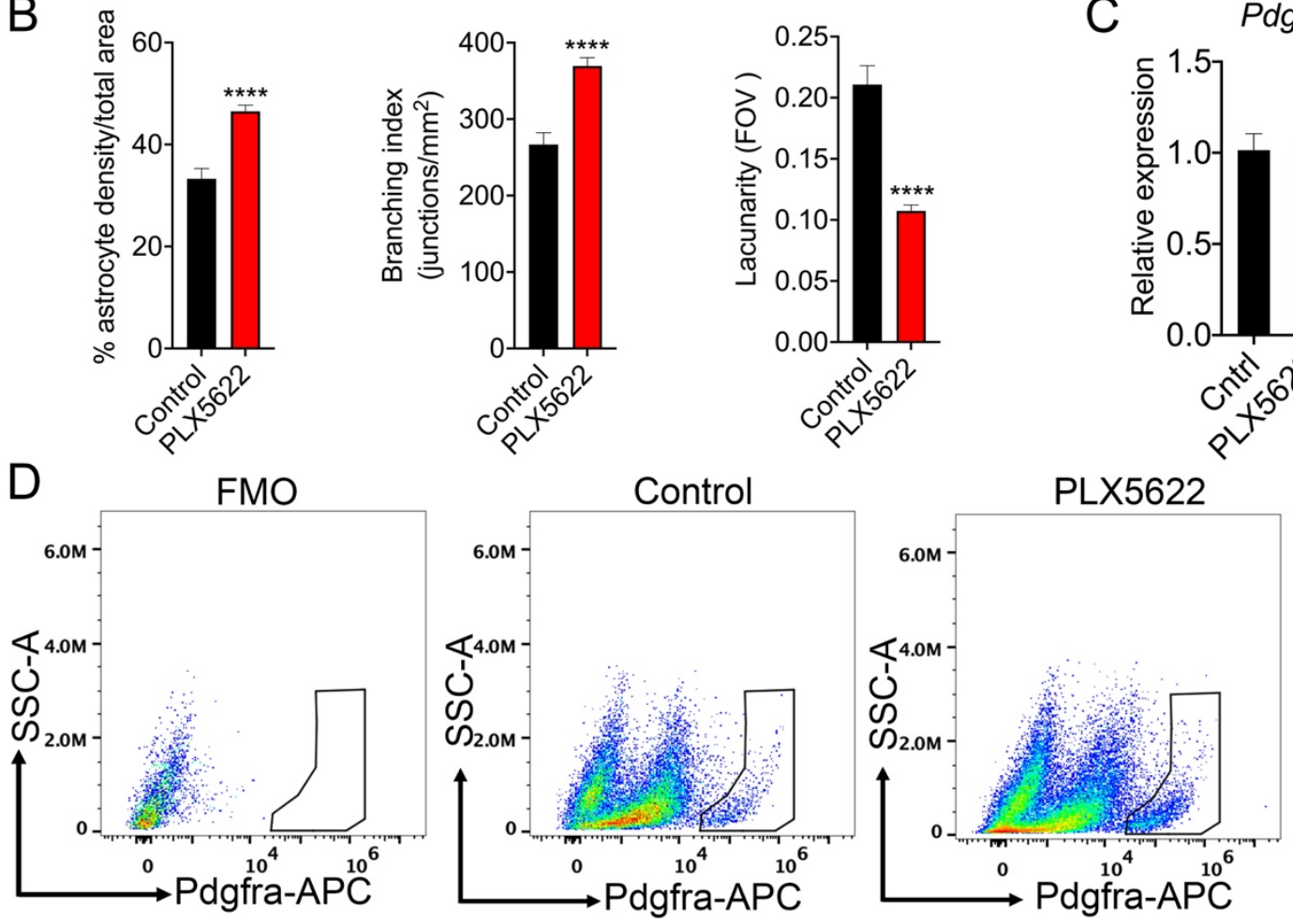

857

858

859

860

\section{1}

862

863

Figure 3. Microglia depletion in early vascular development increases astrocyte density and causes abnormal template formation. Time-pregnant C57BL/6J mice from gestational day E13.5/E14.5 were fed either a control or Csfr1 specific antagonist (PLX5622) diet and littermates were analyzed at P5. (A-E). A. Representative P5 retinal 864 flatmount from control or PLX5622 groups immunostained for Pdgfra and P2ry12. Cell 
866 were observed in avascular central and peripheral retina $(n=4)$. Scale bar: $50 \mu m$. B.

867 Real-time PCR analysis of relative gene expression levels of Pdgfa at P5 $(n=5)$ in the

868 retinas of control or PLX5622 groups. C. Images of control and PLX5622 P5 retinal

869 flatmounts immunostained for Pdgfra in the central region of all four quadrants were

870 acquired. Using 'NIH-ImageJ Angiogenesis Analyzer' software, astrocyte density,

871 branching index, and lacunarity were quantified and shown $(n=5)$. D. Astrocyte (Pdgfra-

$872 \mathrm{APC}+$ ) live cell frequencies were assessed in P5 retinas in either control or PLX5622

873 groups by flow cytometry $(n=3)$. E. Quantification of flow cytometry data (D) showing the 874 percentage of Pdgfra-APC+ live cells in control and PLX5622 groups. All error bars

875 represent \pm S.E.M. Statistical differences between control and PLX5622 group was

876 calculated by unpaired $t$ test. ${ }^{*} \mathrm{P}<0.05,{ }^{* * *} \mathrm{P}<0.001,{ }^{* * *} \mathrm{P}<0.0001$

877

878

879

880

881

882

883

884

885

886 
A CD31-P1

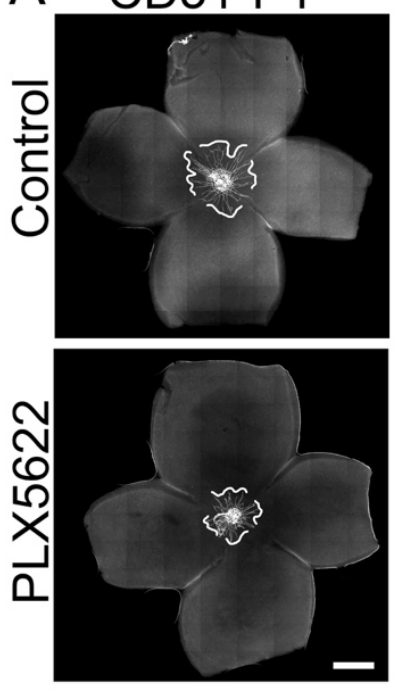

C CD31
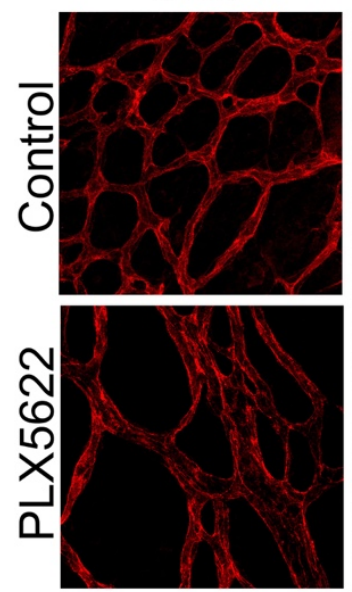

$\mathrm{D}$

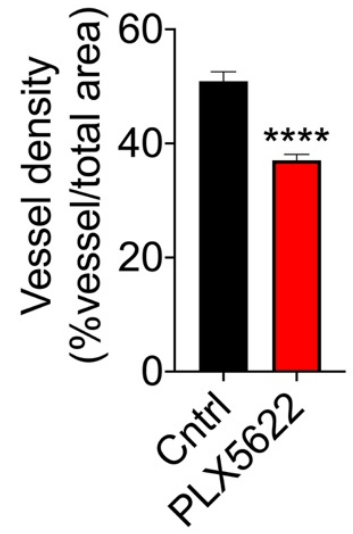

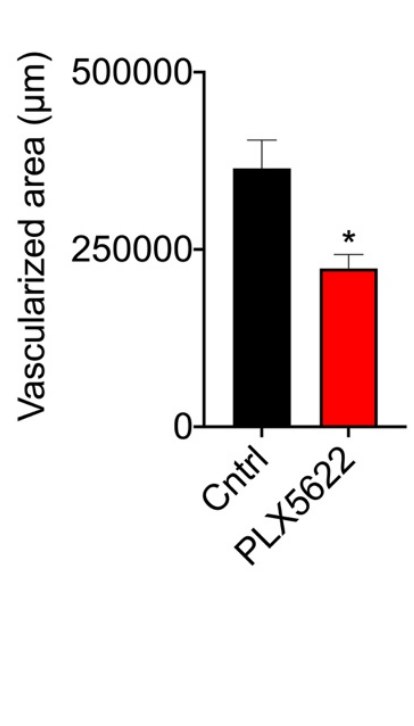

B CD31-P5

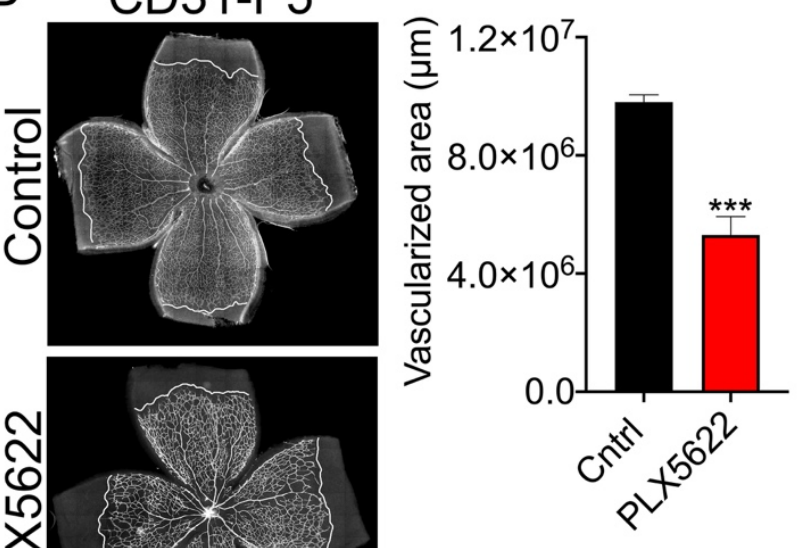

887

888

889

890
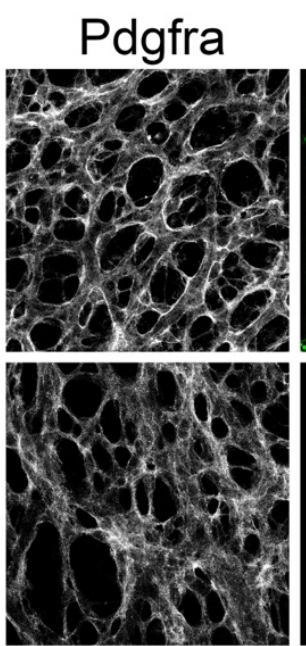

P2ry12
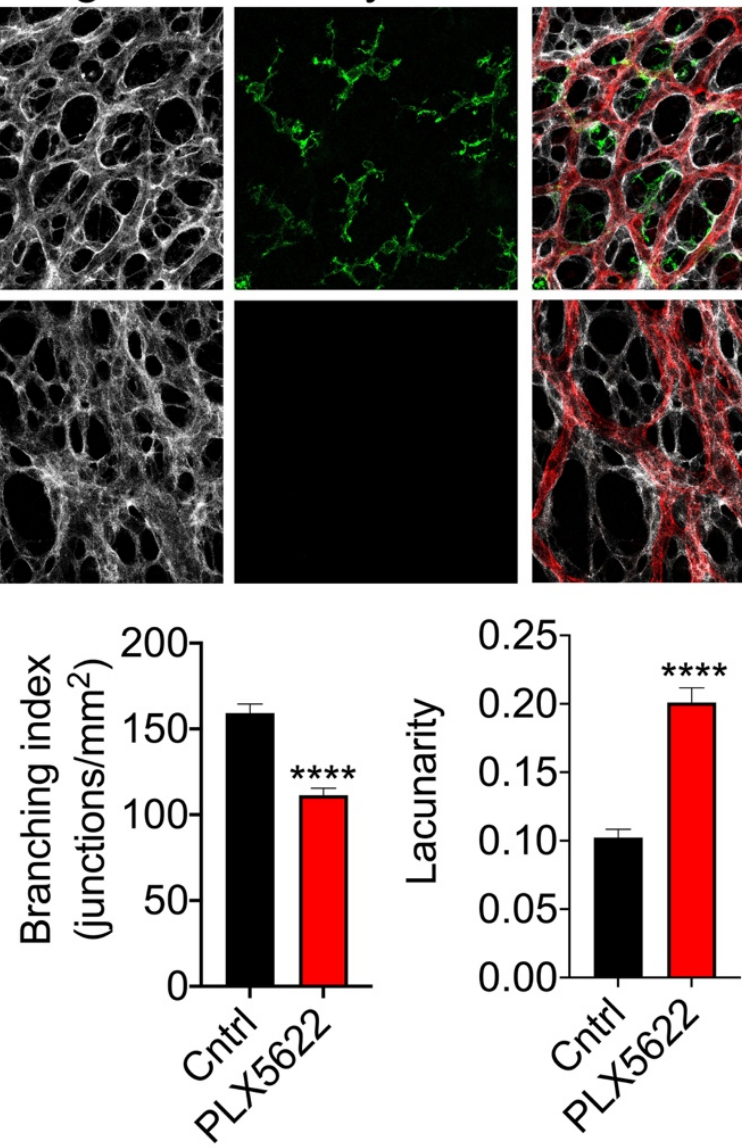

Figure 4. Microglial depletion reduces vascular growth and density. Time-pregnant C57BL/6J mice from gestational day E13.5/E14.5 were fed a control diet or diet containing 
891 Csfr1 specific antagonist (PLX5622) and the littermates were analyzed at respective ages

892 (A-E). A and B. P1 and P5 retinal flatmounts from control and PLX5622 group 893 immunostained for CD31 (endothelial cell marker) and vascularized area (indicated by 894 dotted lines in the images) were quantified and shown in the bar graphs $(\mathrm{n}=4) \mathbf{C}$. 895 Representative images of P5 retinal flatmount from control and PLX5622 group 896 immunostained for CD31, Pdgfra, and P2ry12 showing microglial interaction with 897 endothelial cells and astrocytes in the vascularized area. D. Images of control and 898 PLX5622 P5 retinal flatmounts immunostained for CD31 in the vascularized central region 899 of all four quadrants were acquired. Using 'NIH-ImageJ Angiogenesis Analyzer' software, 900 vessel density, branching index, and lacunarity were quantified and shown $(n=5)$. Scale 901 bars: A and B are $500 \mu \mathrm{m}, \mathrm{C}$ is $50 \mu \mathrm{m}$. All error bars represent \pm S.E.M. Statistical 902 differences between control and PLX5622 group was calculated by unpaired $t$ test. * $\mathrm{P}<$ $0.05,{ }^{* *} \mathrm{P}<0.01,{ }^{* * *} \mathrm{P}<0.001,{ }^{* * * *} \mathrm{P}<0.0001$ 

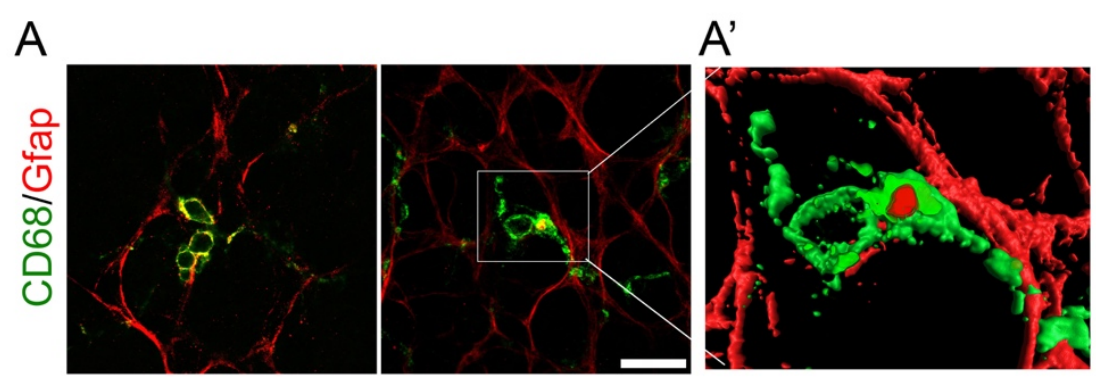

C

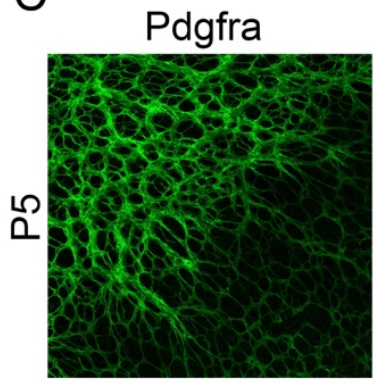

D

Pdgfra/C3-tdT/P2ry12
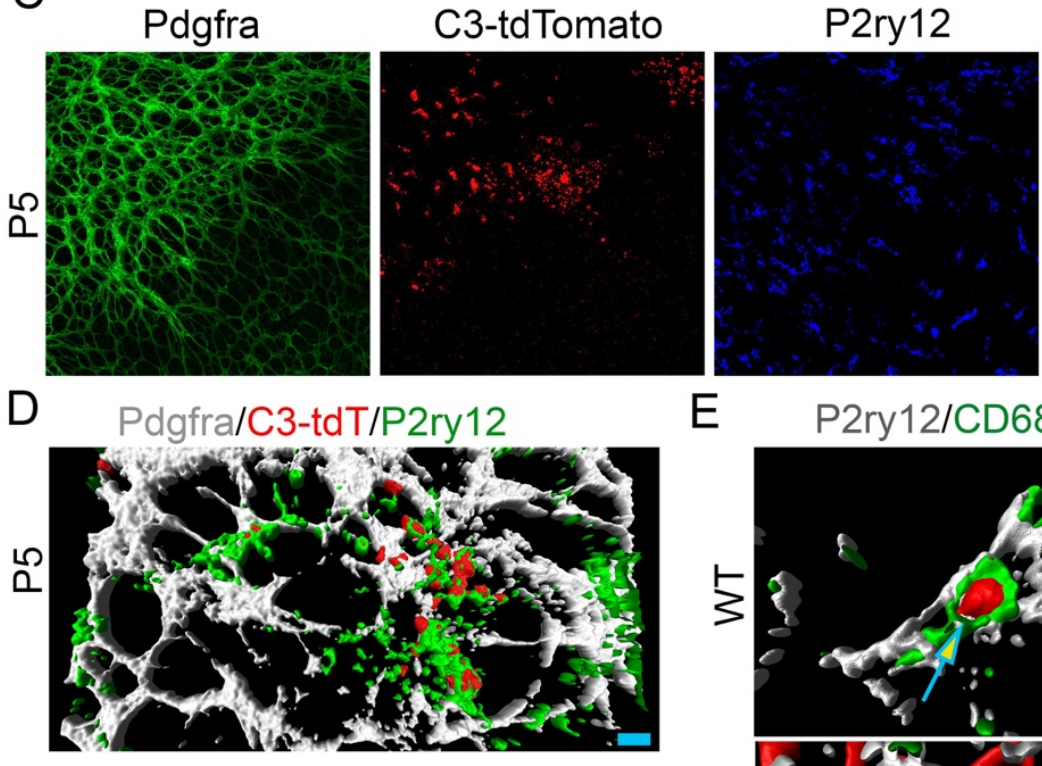

B

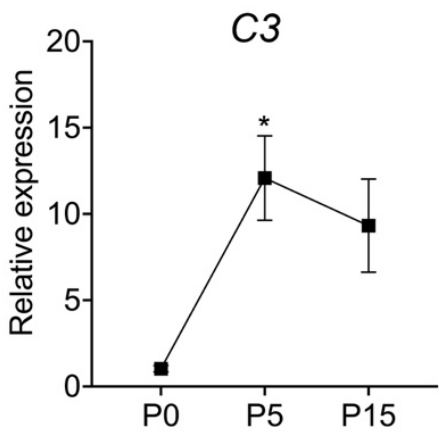

E

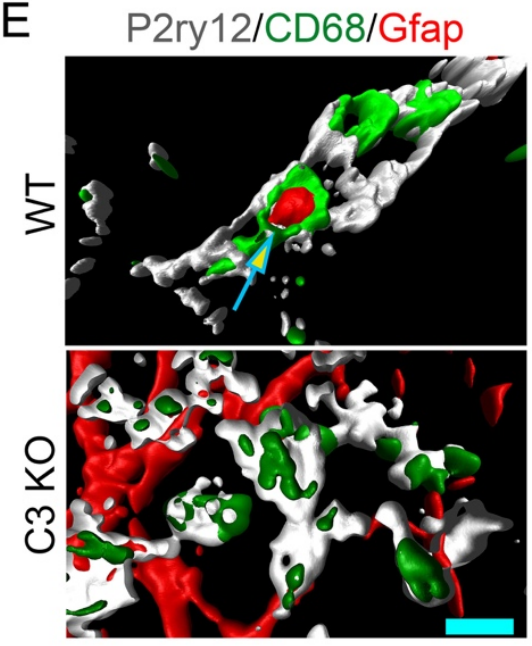

Overlay

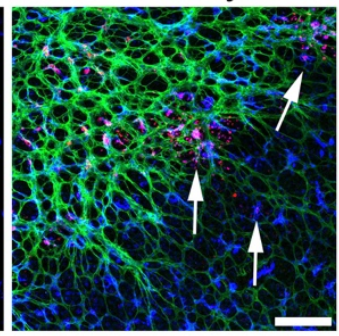

Age
910

911

912

913

914

915

916

917

918

919

920

921

Figure 5. C3 is expressed in microglia and loss of $\mathrm{C} 3$ reduces microglial pruning of astrocytes. A. Representative z-stack images of P5 retinal flatmount showing GFAP and CD68 immunostaining $(n=5)$. $\mathbf{A}^{\prime}$. Enlarged 3D-reconstructed boxed area in $(\mathbf{A})$ revealing microglia internalized GFAP astrocyte debris within CD68+ lysosomes (green). B. Realtime PCR analysis of retinas isolated at P0 (n=3), P5 (n=5), and P15 (n=5) showing age- 
922 dependent relative C3 mRNA levels. C. C3-tdTomato (C3-tdT) expressing P5 retinal

923 flatmount immunostained for P2ry12 and Pdgfra to label microglia (blue) and astrocytes

924 (green) respectively. D. Representative 3D-reconstructed image from C3-tdT expressing

925 P5 retinal flatmount immunostained for P2ry12 and Pdgfra reveal C3 reporter expression

926 within microglia (P2ry12, green) that are closely interacting with astrocytes (Pdgfra, grey)

$927(n=5)$. E. Z-stack images were acquired from P5 WT and C3 KO retinal flatmount

928 immunostained for P2ry12, GFAP, and CD68 and 3D-reconstructed to visualize microglia

929 (P2ry12) engulfed astrocyte (GFAP) bodies within microglial lysosomal/endosomal

930 membrane protein CD68). Bar graph shows the percentage of GFAP debris localized

931 within P2ry12, CD68 co-labelled microglia between WT and C3 KO retinas $(\mathrm{n}=3)$. All error

932 bars represent \pm S.E.M. In B. statistical differences at P0, P5, and P15 were calculated

933 by one-way analysis of variance followed by a post Dunnett's multiple comparisons. * $\mathrm{P}$

$934=0.02$. Statistical differences between WT and C3 KO in E and F was calculated by 935 unpaired $t$ test. ${ }^{*} \mathrm{P}<0.05,{ }^{* *} \mathrm{P}<0.01$. Scale Bars: $\mathrm{A}$ is $15 \mu \mathrm{m}, \mathrm{C}$ is $75 \mu \mathrm{m}, \mathrm{D}$ and $\mathrm{E}$ are $93610 \mu \mathrm{m}$. 


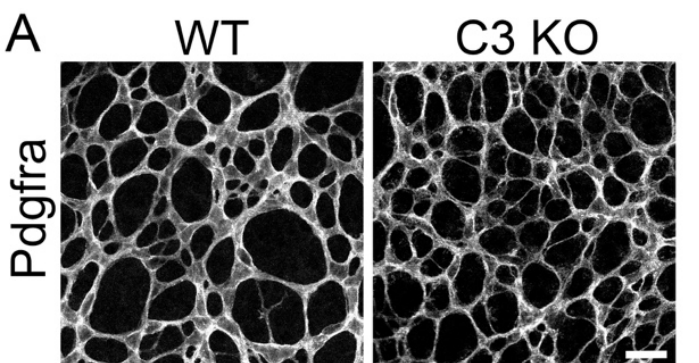

B
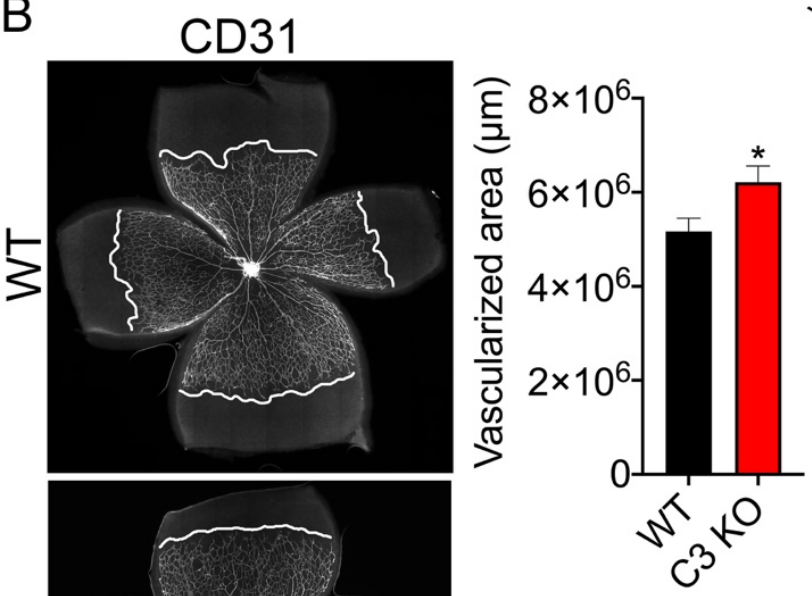

946

947

948

949

950

951

952

953

954 lacunarity using ImageJ Angiogenesis Analyzer software tool $(n=5)$.

B. Representative

955

956

P5 Retinal flatmounts of WT and C3 KO immunostained for CD31 showing vascular

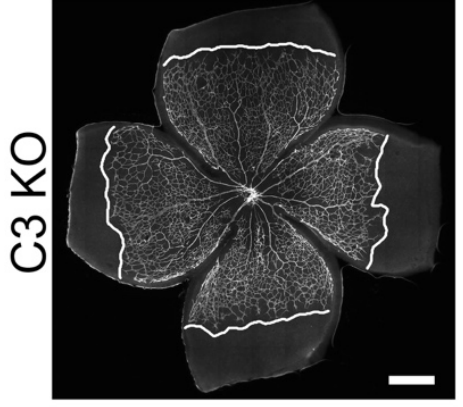

Figure 6. C3 deletion increases astrocytic template and vascular densities at P5. A. showing astrocyte template patterning in the avascular region $(n=5)$. Bar graphs represent the quantitative measurements of astrocyte density, branching index, and$$
\text { P5 Retinal flatmounts of WT and C3 KO immunostained for CD31 }
$$
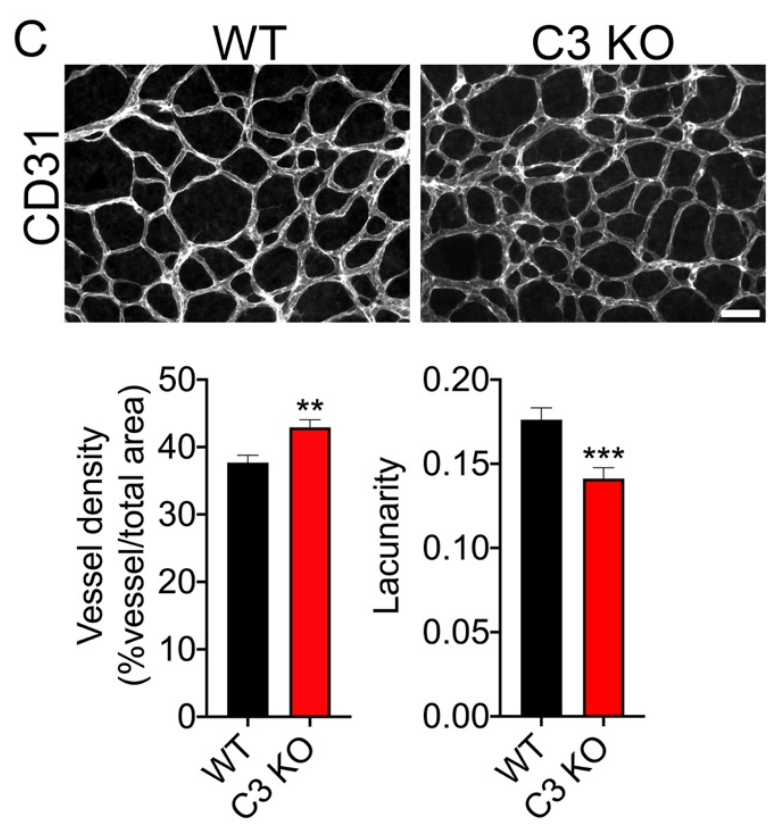
958 the central region to assess vessel density and spatial branching (lacunarity) using

959 'Image J Angiogenesis Analyzer' software tool $(n=5)$. Scale bars: $\mathbf{A}$ is $500 \mu \mathrm{m}, \mathbf{B}$ is $50 \mu \mathrm{m}$,

960 and $\mathbf{C}$ is $20 \mu \mathrm{m}$. All error bars represent \pm S.E.M. Statistical differences between WT and

961 C3 KO was calculated by unpaired $t$ test. ${ }^{* *} \mathrm{P}<0.01,{ }^{* * *} \mathrm{P}<0.001$.

962

963

964

965

966

967

968

969

970

971

972

973

974

975

976 

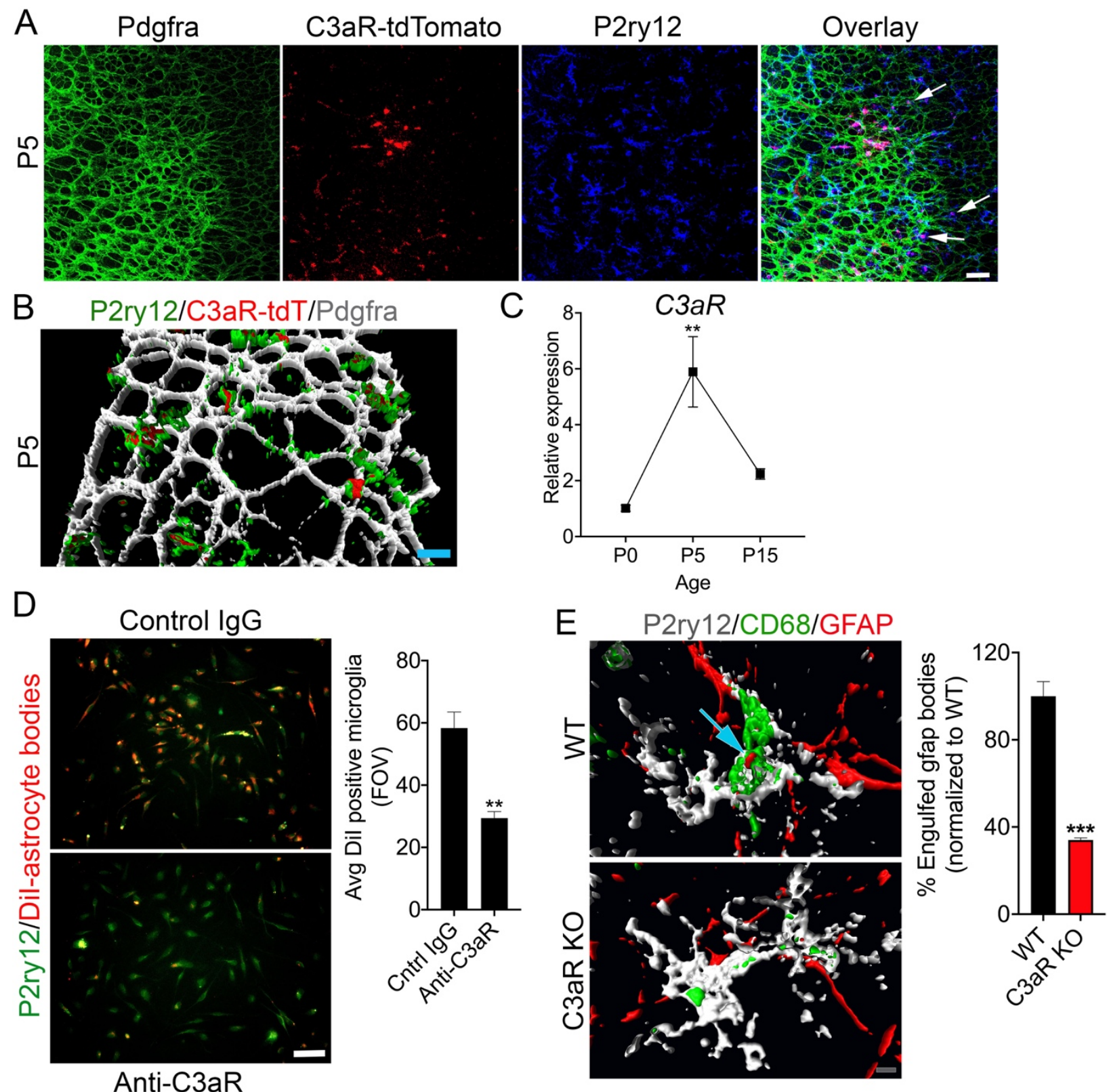

Figure 7. Microglia expresses $\mathrm{C} 3 \mathrm{aR}$ and loss of $\mathrm{C} 3 \mathrm{aR}$ reduces microglial pruning of astrocytes. A. Representative P5 C3aR-tdTomato (C3aR-tdT) expressing retinal

983 flatmount showing Pdgfra and P2ry12 immunolocalization around the vascular growth

984 front $(n=3)$. Arrows indicates the localization of tdTomato in P2ry12 expressing

985 microglia. B. Representative 3D-reconstructed image from C3aR-tdTomato expressing 
986 P5 retinal flatmount immunostained for P2ry12 and Pdgfra reveal C3aR reporter

987 expression within microglia (P2ry12, green) that are closely interacting with astrocytes

988 (Pdgfra, grey) (n=3). C. Real-time PCR analysis of retinas isolated at P0 (n=3), P5

$989(\mathrm{n}=5)$, and P15 $(\mathrm{n}=5)$ showing C3aR relative mRNA levels. D. Primary microglial culture

990 isolated from P5 retina were treated with Dil-labeled retinal astrocyte apoptotic bodies in

991 the presence of control IgG or anti-C3aR IgG for 2 hours and the number of Dil-

992 astrocyte bodies engulfed microglia in the two treatment conditions were quantified and

993 shown in the bar graph $(n=3)$. E. Z-stack images were acquired from P5 WT and C3aR

994 KO retinal flatmount immunostained for P2ry12, GFAP, and CD68 and 3D-

995 reconstructed to visualize microglia (P2ry12) engulfed astrocyte (GFAP) bodies within

996 microglial lysosomal/endosomal membrane protein CD68). Bar graph shows the

997 percentage of GFAP debris localized within P2ry12, CD68 co-labelled microglia

998 between WT and C3aR KO retinas $(n=3)$. All error bars represent \pm S.E.M. Statistical

999 differences at P0, P5, and P15 were calculated by one-way analysis of variance

1000 followed by a post Dunnett's multiple comparisons. ${ }^{*} \mathrm{P}=0.007$. Statistical differences

1001 between IgG control and anti-C3aR IgG was calculated by unpaired $t$ test. Statistical

1002 differences between WT and C3aR KO in E and F were calculated by unpaired $t$ test. **

$1003 \mathrm{P}<0.01,{ }^{* * *} \mathrm{P}<0.001$, and ${ }^{* * *} \mathrm{P}<0.001$. Scale Bars: $\mathrm{A}$ is $75 \mu \mathrm{m}, \mathrm{B}$ and $\mathrm{E}$ is $10 \mu \mathrm{m}, \mathrm{D}$

1004 is $40 \mu \mathrm{m}$,

1005

1006

1007

1008

1009

1010

1011

1012 
A
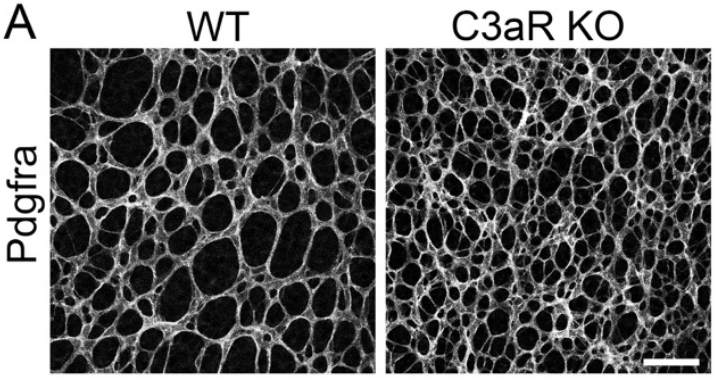

B

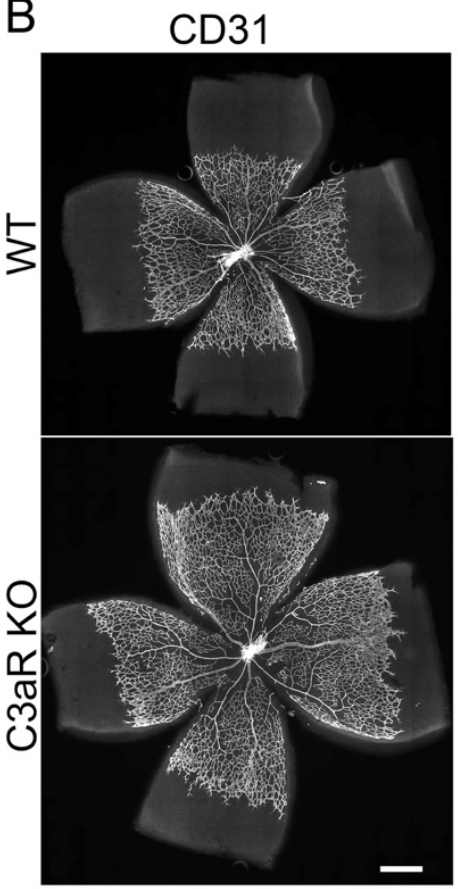

1014

1015

1016

1017

1018

1019

1020

1021

1022

1023
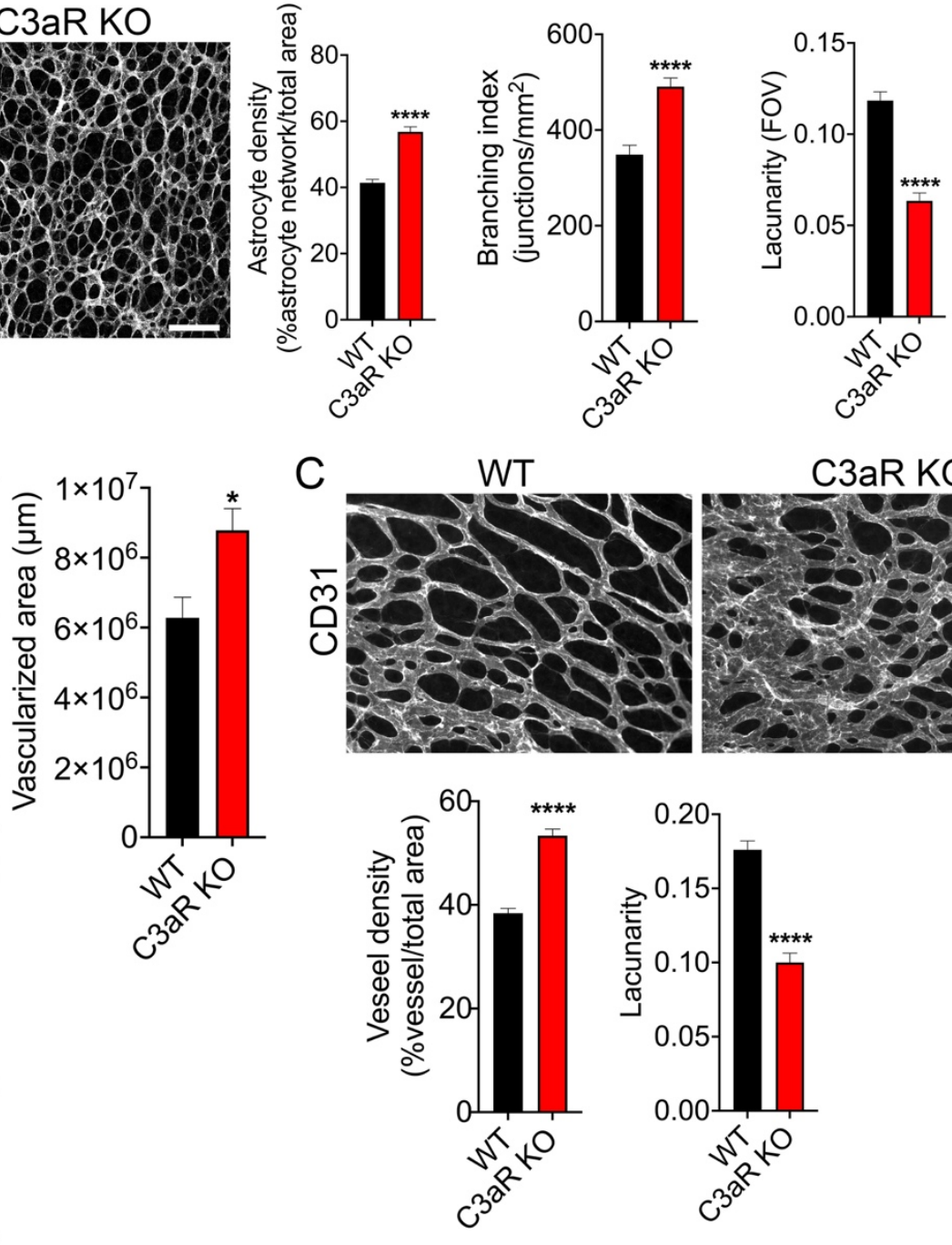
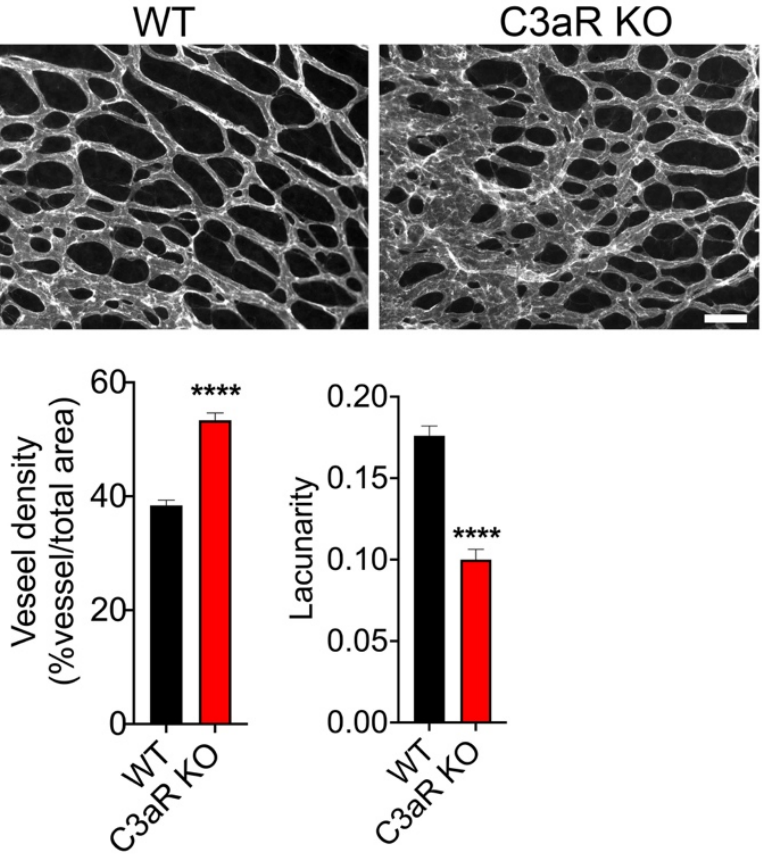

Figure 8. Loss of C3aR increases astrocytic template and vascular densities at P5. A. Representative P5 retinal flatmount from WT and C3aR KO immunostained for Pdgfra showing astrocyte template patterning in the avascular region $(n=5)$. Bar graphs represent the quantitative measurements of astrocyte density, branching index, and lacunarity using ImageJ Angiogenesis Analyzer software tool $(n=5)$.

B. Representative P5 Retinal flatmounts of WT and C3aR KO immunostained for CD31 showing vascular outgrowth and quantification of vascularized area in WT and C3aR KO $(n=5)$. C. P5 retinal flatmounts of WT and C3aR KO were immunostained for CD31 and images were taken 
1024 in the central region (bottom panel) to assess vessel density, and spatial branching 1025 (lacunarity) differences using 'ImageJ Angiogenesis Analyzer' software tool $(n=5)$. Scale 1026 bars: $\mathbf{A}$ is $50 \mu \mathrm{m}, \mathbf{B}$ is $500 \mu \mathrm{m}$, and $\mathbf{C}$ is $50 \mu \mathrm{m}$. All error bars represent \pm S.E.M. Statistical 1027 differences between WT and C3 KO was calculated by unpaired $t$ test. ${ }^{*} \mathrm{P}<0.05$, ${ }^{* * * *} \mathrm{P}$ $1028<0.0001$ 